\title{
Symmetry preference in shapes, faces, flowers and landscapes
}

\author{
Marco Bertamini ${ }^{\text {Corresp., } 1}{ }^{,}$Giulia Rampone ${ }^{2}$, Alexis D.J. Makin ${ }^{1}$, Andrew Jessop ${ }^{3}$ \\ 1 Department of Psychological Science, University of Liverpool, Liverpool, United Kingdom \\ 2 School of Psychology, University of Liverpool, Liverpool, United Kingdom \\ 3 Max Planck Institute for Psycholinguistics, Nijmegen, The Netherlands \\ Corresponding Author: Marco Bertamini \\ Email address: m.bertamini@liverpool.ac.uk
}

Most people like symmetry, and symmetry has been extensively used in visual art and architecture. In this study we compared preference for images of abstract and familiar objects in the original format or when containing perfect bilateral symmetry. We created pairs of images for different categories: male faces, female faces, polygons, smoothed version of the polygons, flowers, and landscapes. This design allows us to compare symmetry preference in different domains. Each observer saw all categories randomly interleaved but saw only one of the two images in a pair. After recording preference, we recorded a rating of how salient the symmetry was for each image, and measured how quickly observers could decide which of the two images in a pair was symmetrical. Results reveal a general preference for symmetry in the case of shapes and faces. For landscapes, natural (no perfect symmetry) images were preferred. Correlations with judgments of saliency were present but generally low, and for landscapes the salience of symmetry was negatively related to preference. However, even within the category where symmetry was not liked (landscapes), the separate analysis of original and modified stimuli showed an interesting pattern: Salience of symmetry was correlated positively (artificial) or negatively (original) with preference, suggesting different effects of symmetry within the same class of stimuli based on context and categorization. 
9 Marco Bertamini $^{1}$, Giulia Rampone ${ }^{2}$, Alexis D.J. Makin ${ }^{1}$, Andrew Jessop ${ }^{3}$

$11{ }^{1}$ Department of Psychological Sciences, University of Liverpool, Liverpool, UK

$12{ }^{2}$ School of Psychology, University of Liverpool, Liverpool, UK

13 39ax Planck Institute for Psycholinguistics, Nijmegen, The Netherlands

20 Correspondence should be sent to

21 Marco Bertamini, The University of Liverpool, Bedford Street South, L69 7ZA Liverpool, UK.

22 E-mail: M.Bertamini@liverpool.ac.uk 
Abstract

24 Most people like symmetry, and symmetry has been extensively used in visual art and

25 architecture. In this study we compared preference for images of abstract and familiar objects in

26 the original format or when containing perfect bilateral symmetry. We created pairs of images

27 for different categories: male faces, female faces, polygons, smoothed version of the polygons,

28 flowers, and landscapes. This design allows us to compare symmetry preference in different

29 domains. Each observer saw all categories randomly interleaved but saw only one of the two

30 images in a pair. After recording preference, we recorded a rating of how salient the symmetry

31 was for each image, and measured how quickly observers could decide which of the two images

32 in a pair was symmetrical. Results reveal a general preference for symmetry in the case of shapes

33 and faces. For landscapes, natural (no perfect symmetry) images were preferred. Correlations

34 with judgments of saliency were present but generally low, and for landscapes the salience of

35 symmetry was negatively related to preference. However, even within the category where

36 symmetry was not liked (landscapes), the separate analysis of original and modified stimuli

37 showed an interesting pattern: Salience of symmetry was correlated positively (artificial) or

38 negatively (original) with preference, suggesting different effects of symmetry within the same

39 class of stimuli based on context and categorization. 

observers tend to prefer the more symmetrical version of a given stimulus, using both familiar objects and abstract patterns (Eisenman, 1967; Rhodes, Proffitt, Grady, \& Sumich, 1998). Some evidence for symmetry as a general aesthetic principle comes from cross cultural studies (for a recent review see Che, Sun, Gallardo, \& Nadal, 2018). In addition to explicit measures, implicit measures have confirmed an association between symmetry and positive valence (Makin, Pecchinenda, \& Bertamini, 2012).

In this study, we examined the preference for symmetry using a deliberately heterogeneous set of images; specifically, faces (males and females), abstract shapes (smooth and angular), flowers, and landscapes. The aim was to compare the role of perfect bilateral symmetry in the context of multiple categories, and to relate preference to rating of the symmetry salience of the items. For one category (landscapes) the symmetry was about positions of objects (composition) and therefore this is a special case of symmetry that is not within an object.

57 selective for processing faces (fusiform face area, FFA), bodies (extrastriate body area, EBA) objects (lateral occipital complex, LOC) and places (parahippocampal place area, PPA), and the fact that these are common elements in works of art (Chatterjee, 2014). This, however, raises the question of whether there are general aesthetic principles, such as preference for symmetry,

61 independent of category, or whether aesthetic principles are specific to each category. 
The early work on symmetry and preference used abstract configurations (e.g. Birkhoff,

63

64

65

66

67

68

69

70

1933; Eisenman, 1967). It is well established that preference for symmetry correlates with the salience of abstract symmetry. Jacobsen and Höfel (2003; Höfel \& Jacobsen, 2007) used black and white patterns and recorded visual evoked potentials. They found preference for reflectional symmetry and strong activation in visual areas. Makin, Helmy and Bertamini (2018) compared preference for abstract patterns where salience had been determined by formal measures of perceptual goodness (van der Helm \& Leeuwenberg, 1996), discrimination speed and amplitude of the visual evoked brain response (Makin et al., 2016). Results were straightforward: measures of salience correlated with preference ratings. The more perceptually obvious the symmetry, and the larger the neural response, the more people liked it. Moreover, the results were similar in samples from UK and Egypt.

Other work has shown that implicit preference for symmetry is closely related to how quickly symmetry can be discriminated (e.g. Makin et al., 2012; Makin, Pecchinenda, \& Bertamini, 2012). Despite this strong link, it is also known that preference for symmetry varies with several factors, in particular with age and sex (Humphrey, 1997) and with training (Eysenck \& Castle, 1970). Although, importantly, symmetry preference is stable in the context of category learning (Rentschler, Jüttner, Unzicker, \& Landis, 1999). Moreover, McManus (2005) has argued that there is always a tension between symmetry and asymmetry, in the sense that the best balance may not be achieved by perfect symmetry.

The literature on the attractiveness of faces is vast (for a review, see Rhodes, 2006). The universality of this preference in humans is supported by cross-cultural and developmental studies (e.g., Perrett, May, \& Yoshikawa, 1994). Symmetry contributes to beauty for both males and females faces, with evolutionary psychologists highlighting the role of symmetry in 
85 signalling mate quality and health (e.g., Gangestad \& Thornhill (1997); Watson \& Thornhill

86 (1994). Evidence in support of the link between facial symmetry and health is mixed (for a

87 negative finding see Pound et al., 2014) and even chicken prefer more symmetrical human faces

88 (Ghirlanda, Jansson \& Enquist, 2002). There is an alternative position that argues that preference

89 for symmetry is a by-product of how information is processed by neural systems (Enquist \&

90 Arak, 1994; Enquist \& Johnstone, 1997). This alternative view has been highlighted recently by

91 Ryan: "“preferences for symmetry in sexual traits may have nothing to do with good genes of the

92 courter but more with how the brains of the choosers work" " (Ryan, 2018, p.68). However, it

93 should be noted that these two positions are not mutually exclusive.

Little (2014) has argued that symmetry preference is domain specific and stronger in the case of faces. This is consistent with an evolutionary view claiming that symmetry is an index of the ability of an organism to cope with developmental stress and thus an index of mate quality. In

97 support of this view it has been reported that preference for symmetry in faces is stronger for upright than for inverted faces (Little \& Jones, 2003). The study by Little (2014) compared responses to human faces, primate faces, and abstract art images. They reported strongest

100 preference for symmetry in human faces. However, the faces were discrete objects while the 101 abstract patterns used were patches of colour that did not form a single object. It is known that

102 symmetry is more salient in the case of other grouping factors (i.e., within rather that between 103 objects; e.g. Bertamini, 2010). Recently, Vessel, Maurer, Denker and Starr (2018) found strong

104 inter-individual agreement (i.e., shared taste) for faces and to a lesser extent for landscapes, and

105 low agreement for architecture and in particular for artworks. This suggests the possibility that

106 there are different mechanisms, and different factors affecting symmetry preference for different

107 categories of objects. For faces symmetry may be linked to attractiveness, and possibly mate 
108 quality. At the other extreme we have bilateral symmetry between objects, such a physical layout

109 of different objects, it is possible that symmetry is not a predictor of preference.

$110 \quad$ Flowers are interesting stimuli for different reasons. The growth process may explain

111 some of the symmetry present in flowers, just as in leaves and other biological features. In

112 addition, flowers have evolved to be salient visual stimuli and attractive to insects in particular,

113 as this is their function. Bumblebees for example show an innate preference for bilateral

114 symmetry in flowers (Rodríguez et al., 2004). In a recent study, Hůla and Flegr (2016) asked

115 observers to rate the beauty of 52 common wildflowers. Flower with radial symmetry and low

116 complexity were rated as the most beautiful. The authors note that these are more prototypical as

117 flowers. There was also a preference for more coloured flowers (in particular for blue). Although

118 most flowers possess a degree of symmetry, just as for human faces, this symmetry is not

119 perfect. It is possible therefore to modify the images to create flowers with perfect bilateral

120 symmetry.

121 Landscapes have been studied in terms of preference, although in some cases they have

122 been selected as rich stimuli but without a specific interest in what makes landscapes special.

123 Rapid categorization of scenes is possible, for example in terms of openness, and is probably

124 based on coarsely localized information (Oliva \& Torralba, 2001) and colour (Oliva \& Schyns,

125 2000). With respect to preference, the biophilia hypothesis suggests that preference for open

126 space with sparse vegetation and water may reflect the characteristics of the environment

127 familiar to our common African ancestors (Wilson, 1984). In one study, Falk and Balling (2010)

128 found evidence for this hypothesis by presenting participants with diverse types of landscapes

129 (see also Orians \& Heerwagen (1992)). Using functional magnetic resonance imaging, Yue,

130 Vessel and Biederman (2007) found that viewing scenes independently rated as preferred, was 
131 associated with greater activation in the parahippocampal cortex, but not in the lateral occipital

132 complex (LOC). As in other studies, the category scenes included a variety of settings, some

133 outdoors and some indoors. For the purpose of our study by landscape we mean an outdoor scene

134 with views extending in the distance.

135 Landscapes are unlike single objects in important ways. A body that is subject to growth

136 will contain some degree of symmetry, but a natural landscape is shaped by multiple physical

137 processes. It is unlikely that hills, boulders or trees will be located in a symmetrical pattern. This

138 can be contrasted with non-natural, highly constrained scenes of towns or gardens. Our study is

139 therefore the first that allows a direct comparison of the strength of preference for symmetry in

140 landscapes as compared to other categories. As noted above there are good reasons to expect

141 symmetry to be closely associated with objectness, and therefore to have a different function

142 within rather than between objects.

The current study

144 We selected images of faces (males and females), abstract shapes (smooth and angular),

145 flowers, and landscapes. Observers were asked for ratings of beauty in the context of multiple

146 categories. For each item we had two versions, one had perfect bilateral symmetry and the other

147 had a much lower degree of regularity. We will us the terms original and bilateral symmetry

148 respectively. Examples of the stimuli are provided in Figure 1. The images as well as the raw

149 data are available on Open Science Framework (osf.io/9qz6p).

150 Each observer, however, never saw both versions of a given item. Perfect symmetry is

151 rare in nature, and there is some evidence in the case of faces that when it appears artificial then

152 less symmetrical faces may be preferred in some cases (Zaidel \& Hessamian, 2010). Therefore, 
153 although we predict a preference for symmetry, preference may depend both on its salience (how

154 easy it is to see) and on how artificial it may appear.

155 In a second phase of the study observers rated how symmetrical each image appeared

156 (rating of symmetry salience) and in a third phase they discriminated which of the pair was the

157 symmetrical version as quickly as possible (response time was recorded). The aim was to test

158 preference for symmetry in a context of multiple categories, without drawing attention to

159 symmetry as the key variable. We will analyse the link between preference and observer ratings

160 of the salience of the symmetry in the pattern. This correspondence could provide support for the

161 view that preference is linked to the tuning of the visual system (Enquist \& Arak, 1994;

162 Ramachandran \& Hirstein, 1999; Redies, 2007).

163 People may like what they find easy to perceive, providing a direct link between

164 perception and emotion. Leder, Belke, Oebert and Augustin (2004) formulated a model of visual

165 aesthetic judgment, where symmetry is placed, among other factors, within the early perception

166 analysis stage. Symmetry is an optimal stimulus for the human visual system to process, and

167 observers may like it for this reason. Alternatively, the link between preference and fluency may

168 work in a more indirect way because of the positive associations with fluency of processing. This

169 latter idea is known as fluency hypothesis (Reber, Schwarz, \& Winkielman, 2004). A recent

170 proposal is that fluency as an amplification role, in the sense that it amplify the pre-existing

171 valence of a stimulus (Albrecht \& Carbon, 2014).

172 Despite the extensive literature on preference for symmetry, the relationship between

173 salience and preference for symmetry in different domains requires more exploration. There is no

174 guarantee that preference in one domain will generalize to other domains (Makin, 2017). 


\section{Participants}

178 Forty-two individuals (8 male, 34 female) took part in the study and were recruited from

179 the University of Liverpool student community. The age range was 18-24 and six were left

180 handed.

181

182 Stimuli

There were six sets of ten pairs of images. For each set, ten images were the original versions (partly symmetrical but without perfect vertical bilateral reflection) and ten images had perfect vertical symmetry. The sets were: male faces, female faces, angular shapes, smooth shapes, flowers, landscapes. All images were in greyscale. We used a black background and the images appeared within a square region of 500 pixels, except for the landscapes that had a height of 500 pixels and a width of 800 pixels.

190 for symmetry: Rhodes, Proffitt, Grady, and Sumich (1998). There were ten males and ten

191 females faces, either as veridical images of human faces or as manipulated images to contain

192 perfect bilateral symmetry, thus generating new images of the ten individuals.

Abstract shapes: Points were selected from a circle to create a polygon. For more details

194 on the procedure, see Palumbo, Ruta and Bertamini (2015) and Bertamini, Palumbo, Gheorghes

195 and Galatsidas (2016). Around the polygon there were always 20 convex and 16 concave

196 vertices (total 36). The radius of the underlying circle could vary randomly by $54 \%$ in length

197 making the polygon irregular. Symmetrical versions were created by using the left side of the

198 polygon and setting the vertices on the right side by reflection. There were two categories of 
199 abstract shapes, one was the original polygons and we refer to these stimuli as Angular (ten

200 pairs). A second set was created by using a cubic spline that made the contour smooth. We refer

201 to this set as Smooth (ten pairs).

202 Flowers: the flower stimuli were from taken from a study by Hůla and Flegr (2016). In

203 this study, people rated 52 flowers for beauty. Results found a preference for blue colour and for

204 radially symmetrical flowers. We selected the following species: Epipactis palustris; Euphrasia

205 rostkoviana; Impatiens noli-tangere; Lathyrus tuberosus; Limodorum abortivum; Melittis

206 melissophyllum; Mimulus moschatus; Ophrys apifera; Pisum sativum; Tropaeolum majus;

207 Veronica beccabunga; Viola biflora; Viola reichenbachiana. Starting from the original images we

208 removed colour and manipulated symmetry to obtain ten pairs of images. One set had the

209 original image of the flower and the other had perfect bilateral symmetry.

210 Landscapes: Images of outdoor scenes were downloaded from the internet. We used the

211 keyword "landscape" on Google image search with a setting of "free to use share or modify".

212 Starting from the original images we removed colour and manipulated vertical symmetry to

213 obtain ten pairs of images. As for the other categories, one set had the original image of the

214 landscape and the other had perfect bilateral symmetry.

215 In summary, for all stimuli (with exception for the abstract shapes) symmetry

216 corresponded to an artificial manipulation, as opposed to the original, natural version. This

217 allowed us to test whether the presence of perfect symmetry would automatically predict higher

218 preference, even in objects - and arrangements of objects - that are not perfectly symmetrical by

219 nature. 
220 Procedure

221 The experiment had approval from The Health and Life Sciences Research Ethics

222 Committee (Psychology, Health and Society) at the University of Liverpool (Ref: Bertamini:

223 0540). All participants were given information and signed a consent form before the start of the 224 study.

225 Each participant was tested individually in a quiet room. They completed three phases

226 always in the same order. First participants rated the beauty of each image using a mouse to

227 control a rating scale $(1=$ not at all, $10=$ very beautiful $)$. Second, they used a similar rating scale

228 to rate how "clear" and "salient" the symmetry was in the images $(1=$ not at all, $10=$ very

229 salient). The question was phrased as follows: "You will be presented with the same images

230 again. You will need to indicate how 'obvious' or 'salient' is the symmetry in each image (from

231 not at all at very salient)".

232 The last phase had a different format, participants saw a pair of images and selected the 233 more symmetrical of the two. They were asked to respond with one of two keys ('a' and ' 1 ') as

234 quickly as possible. The presentation of the stimuli and the recording of the responses was

235 controlled by a program in Python using the PsychoPy software (Peirce, 2007). Distance from

236 the screen was not enforced, but at a natural distance of approximately $57 \mathrm{~cm}$ all stimuli were 10

237 deg of visual angle in height. The three tasks are shown in Figure 2.

238 There were two versions of the procedure. We had ten pairs of images for each category, 239 one original and one with bilateral symmetry. Therefore, 60 images were selected so that for

240 each category there were five original and five bilaterally symmetrical items. These 60 items

241 were used for one version (A) of the experiment. In another version (B) the total number was the

242 same but for each item we used the symmetrical image (if the original was used in set A) and 
243 vice versa. This ensured that for each participant a given face, flower and so on was only

244 presented in one version (either original or symmetrical). This difference between set A and B

245 applies to rating of preference and salience, and not for the discrimination task in which the pair

246 was presented on the screen. Upon completion, participants were asked whether or not they had

247 any strategy throughout the study.

249 Analysis

In all the analyses, we split the categories into groups. We tested the results for faces by including male and female as a category. Similarly, we tested the results for abstract shapes by including angular and smooth as a category. This is because we were interested in sex and in angularity as possible factors affecting preference. We did not have similar factors for flowers 254 and for landscapes.

All of the analyses in the present work used generalised linear mixed-effects models 256 implemented in the lme4 package (Bates, Mächler, Bolker, \& Walker, 2015) in $R$ version 3.3.3 (R Core Team, 2018). The dependent measures of these analyses were subjective ratings of beauty or symmetry, or the response times in the discrimination task. A standardisation procedure was applied to these scores, which involved subtracting the mean from the individual ratings, and then dividing each score by the standard deviation. The mean and standard deviations used to standardise the scores were derived from each individual participant rather

262 than the whole sample. All models included symmetry (symmetric/original) as an effect coded 263 fixed factor. Stimulus type was also included in the analyses for faces (male/female) and abstract

264 shapes (angular/smooth), but not for the flower or landscape analysis. The initial random effects 265 structure represented the maximal model (Barr, Levy, Scheepers, \& Tily, 2013). For all analyses, 
266 we included participant as a random intercept, together with item (the different images) and

267 version (set A and set B), each with fully crossed random slopes for symmetry and stimulus type.

268 We have no theoretical interest in these variables and we treat them therefore as random factors.

269 The model was simplified until convergence was reached where necessary. Log likelihood-ratio (

$270 \chi^{2}$ ) comparisons were obtained through the sequential decomposition of the model (Bates et al.,

271 2015), which provided confirmatory tests for the predictors. The marginal and conditional $R^{2}$

272 effect sizes are also reported as measures of the variance explained by the model with the

273 random effect structure included (conditional $R^{2}$ ) and excluded (marginal $R^{2}$ ) from the

274 calculation (Johnson, 2014; Nakagawa \& Schielzeth, 2013; Nakagawa, Johnson, \& Schielzeth, 275 2017).

276

277

Results

278

279

\section{Preference (beauty ratings)}

Before standardisation, beauty score was highest for landscapes $(\mathrm{M}=74.813 ; \mathrm{SE}=1.046)$

280

and lowest for angular shapes $(\mathrm{M}=30.629 ; \mathrm{SE}=0.884)$. For smooth shapes it was 33.737

281 ( $\mathrm{SE}=0.942)$. For males and females faces it was respectively: 32.724, 42.993 ( $\mathrm{SE}=0.85,0.971)$.

282 Finally, for flowers it was $51.578(\mathrm{SE}=1.1)$.

283

Similarly, the standardised beauty score was highest for landscapes $(M=1.343 ; S E=0.043)$

284 and lowest for angular shapes $(M=-0.622 ; S E=0.036)$. For smooth shapes it was -0.476

$285(S E=0.038)$. For males and females faces it was respectively: $-0.498,-0.059(\mathrm{SE}=0.036,0.04)$.

286 Finally, for flowers it was 0.285 ( $\mathrm{SE}=0.047)$. Mean values are plotted in Figure 3.

The maximal model that converged for the faces stimuli contained random intercepts for participant, item, and version, but no random slopes. The model revealed that the standardised 
289 beauty ratings for faces with perfect bilateral symmetry were significantly higher than for 290 original faces $\left(\beta=0.0462, S E=0.0202, \chi^{2}=4.26, p=.039\right)$. Furthermore, there was a

291 significant effect of stimulus type $\left(\beta=-0.2216, S E=0.0202, \chi^{2}=110.7, p<.001\right)$, as higher

292 ratings of beauty were given to female faces than male faces. There was also a marginal

293 interaction between symmetry and stimulus type $\left(\beta=-0.0354, S E=0.0202, \chi^{2}=3.06, p=.080\right)$,

294 as a larger decrease in beauty ratings were observed for original female faces than original male

295 faces, compared to their symmetric counterparts. This model accounted for $10.31 \%$ of the

296 variance in the data without the random-effects, but $38.93 \%$ when they were included $\left(R_{m}^{2}=\right.$

$\left.297 \quad 0.1031 ; R_{c}^{2}=0.3893\right)$.

The maximal model that converged for abstract shapes also only included the random

299 intercepts for participant, item, and version. This revealed that the standardised beauty ratings

300 were significantly higher for symmetrical than original shapes $\left(\beta=0.1399, S E=0.0188, \chi^{2}=\right.$

$30152.09, p<.001)$. Furthermore, there was a significant effect of stimulus type $(\beta=-0.0764, S E=$

$\left.3020.0188, \chi^{2}=16.34, p<.001\right)$, as higher ratings of beauty were given to smooth shapes than

303 angular shapes. There was no interaction between symmetry and stimulus type $(\beta=-0.0114, S E$

$304=0.0188, \chi^{2}=0.37, p=.543$ ). This model accounted for $5.37 \%$ of the variance in the data

305 without the random-effects, but $40.01 \%$ when they were included $\left(R_{m}^{2}=0.0537 ; R_{c}^{2}=0.4001\right)$.

306 In the case of flowers, the maximal model consisted of the random intercepts for

307 participant, item, and version. This model did not confirm higher beauty ratings for symmetrical

308 flowers $\left(\beta=-0.0155, S E=0.0357, \chi^{2}=0.19, p=.664\right)$, and accounted for $0.03 \%$ of the variance

309 in the data without the random-effects, and $32.51 \%$ when they were included $\left(R_{m}^{2}=0.0003 ; R_{c}^{2}\right.$ 
$310=0.3251)$. It is worth noting that in the original study by (Hůla \& Flegr, 2016), beauty was

311 mainly linked with radial symmetry of flowers and not bilateral symmetry.

312 The maximal model for the landscapes data also consisted of random intercepts for

313 participant, item, and version. Conversely, this analysis data showed higher beauty ratings for the

314 original images $\left(\beta=-0.0845, S E=0.0299, \chi^{2}=7.93, p=.005\right)$ and explained $1.06 \%$ of the

315 variance in the data without the random intercepts and $45.88 \%$ when they were included $\left(R_{m}^{2}=\right.$

$\left.3160.0106 ; R_{c}^{2}=0.4588\right)$.

318 Salience (symmetry ratings)

319 Ratings of salience of symmetry were analysed in the same way as ratings for beauty. In

320 all of the models, the maximal random-effects structure that converged included the random

321 intercepts of participant, item, and version, with no random slopes.

322

The non-standardised salience score was highest for flowers (mean=66.875; $\mathrm{SE}=1.736)$

323

and lowest for smooth shapes (mean=51.496; $\mathrm{SE}=2.068$ ). For angular shapes it was 51.924

324 ( $\mathrm{SE}=2.023)$. For males and females faces it was respectively: 58.247, 61.344 ( $\mathrm{SE}=1.708,1.682)$.

325 Finally, for landscapes it was $58.906(\mathrm{SE}=2.035)$. Mean values are plotted in Figure 4.

326 The standardised salience score was highest for flowers (mean=0.267; $\mathrm{SE}=0.05)$ and

327 lowest for smooth shapes (mean=-0.216; $\mathrm{SE}=0.06)$. For angular shapes it was $-0.21(\mathrm{SE}=0.059)$.

328 For males and females faces it was respectively: $-0.001,0.089$ ( $\mathrm{SE}=0.049,0.047)$. Finally, for

329 landscapes it was $0.036(\mathrm{SE}=0.06)$.

330 The model for faces revealed that the standardised salience ratings were significantly

331 higher for symmetric faces than original faces $\left(\beta=0.6382, S E=0.0195, \chi^{2}=646.94, p<.001\right)$.

332 Furthermore, there was a significant effect of stimulus type $\left(\beta=-0.0468, S E=0.0195, \chi^{2}=5.9\right.$, 
$333 p=.015)$, as higher ratings of symmetry were given to female faces than male faces. There was

334 also an interaction between symmetry and stimulus type $\left(\beta=0.0645, S E=0.0195, \chi^{2}=10.9, p\right.$

$335<.001)$, because there was little difference in salience for male and female faces when they were 336 symmetrical, but a larger difference for the original stimuli. This is reasonable given that there

337 was more variability in symmetry in the original faces. This model accounted for $56.73 \%$ of the

338 variance in the data without the random-effects, and $60.53 \%$ when they were included $\left(R_{m}^{2}=\right.$

$\left.0.5673 ; R_{c}^{2}=0.6053\right)$.

The maximal model for abstract shapes revealed that the standardised beauty ratings were

341 significantly higher for symmetrical than original shapes $\left(\beta=0.9511, S E=0.0171, \chi^{2}=\right.$

$3421239.09, p<.001)$. There was no significant effect of stimulus type $\left(\beta=-6 \mathrm{e}-04, S E=0.0171, \chi^{2}\right.$

$343=1.6 \mathrm{e}-03, p=.968)$ and no interaction between symmetry and stimulus type $(\beta=-0.0204, S E=$

$3440.0171, \chi^{2}=1.42, p=.233$ ). This model accounted for $77.21 \%$ of the variance in the data

345 without the random-effects, but $79.96 \%$ when they were included $\left(R_{m}^{2}=0.7721 ; R_{c}^{2}=0.7996\right)$.

346 In the case of flowers, the model confirmed higher symmetry salience ratings for

347 symmetrical flowers $\left(\beta=0.6227, S E=0.0279, \chi^{2}=309.63, p<.001\right)$. This model accounted for

$34849.74 \%$ of the variance in the data without the random-effects, and $63.11 \%$ when they were

349 included $\left(R_{m}^{2}=0 ; R_{c}^{2}=0.6311\right)$.

350 Finally, in the case of landscapes the model revealed higher symmetry salience ratings for

351 symmetrical images $\left(\beta=0.997, S E=0.0218, \chi^{2}=717.41, p<.001\right)$. This model accounted for

$35281.92 \%$ of the variance in the data without the random-effects, and $83.86 \%$ when they were

353 included $\left(R_{m}^{2}=0.8192 ; R_{c}^{2}=0.8386\right)$. 
In summary, for all categories more symmetrical images were rated as more symmetrical.

355 People produced ratings of symmetry salience also for images that were perfectly symmetrical.

356 This variability reflects subjective evaluation of symmetry or possible additional symmetry

357 present independently of the bilateral symmetry manipulation.

\section{Speeded Discrimination}

360

The response time necessary to discriminate which of two images was symmetrical was

analysed in the same way as ratings for beauty and salience. The only important difference is that

362 here the factor Position refers to the order of the images in the pair. Position symmetry means

363 that symmetry was on the left (and original on the right) and Position original means that original

364 was on the left (and symmetry on the right). The maximal model that converged contained

365 random intercepts for participant, item, and version, with no random slopes.

366 Before standardisation, response time was fastest for smooth shapes (mean=0.888;

$367 \mathrm{SE}=0.017$ ) and slowest for female faces (mean=1.294; $\mathrm{SE}=0.037)$. For angular shapes it was

$3680.893(\mathrm{SE}=0.019)$. For males faces it was $1.23(\mathrm{SE}=0.037)$. For landscapes it was 1.041

$369(\mathrm{SE}=0.017)$ and for flowers it was $1.078(\mathrm{SE}=0.026)$. Similarly, the standardised response time

370 was fastest for smooth shapes (mean=-0.231; $\mathrm{SE}=0.046$ ) and slowest for female faces

371 (mean=0.304; $\mathrm{SE}=0.067)$. For angular shapes it was $-0.229(\mathrm{SE}=0.05)$. For male faces it was

$3720.066(\mathrm{SE}=0.054)$. For landscapes it was $0.068(\mathrm{SE}=0.049)$ and for flowers it was 0.046

373 (SE=0.058). Mean values are plotted in Figure 5.

374 The model revealed that there was no difference in response time when the symmetrical

375 image was presented to the left or to the right $\left(\beta=0.0263, S E=0.0388, \chi^{2}=0.45, p=.503\right)$.

376 There was a significant effect of stimulus type $\left(\beta=-0.1187, S E=0.0388, \chi^{2}=9.36, p=.002\right)$, 
377 as faster responses were given to male faces than female faces. There was no interaction between

378 symmetry and stimulus type $\left(\beta=0.0308, S E=0.0388, \chi^{2}=0.63, p=.426\right)$. This model

379 accounted for $1.35 \%$ of the variance in the data without the random-effects, and $1.73 \%$ when

380 they were included $\left(R_{m}^{2}=0.0135 ; R_{c}^{2}=0.0173\right)$.

381 The model for abstract shapes revealed that there were faster responses when the

382 symmetrical image was presented to the left $\left(\beta=0.0856, S E=0.0306, \chi^{2}=7.79, p=.005\right)$.

383 There was no significant effect of stimulus type $\left(\beta=5.30 \mathrm{e}-05, S E=0.0306, \chi^{2}=1.1 \mathrm{e}-05, p=\right.$

$384.997)$ and no interaction $\left(\beta=0.0471, S E=0.0306, \chi^{2}=2.38, p=.123\right)$. This model accounted

385 for $1.22 \%$ of the variance in the data without the random-effects, but $4.03 \%$ when they were

386 included $\left(R_{m}^{2}=0.0122 ; R_{c}^{2}=0.0403\right)$.

387

In the case of flowers, the model did not confirm any difference whether symmetry was

388 on the left or the right $\left(\beta=0.0079, S E=0.0513, \chi^{2}=0.02, p=.878\right)$. This model accounted for

$389<0.01 \%$ of the variance in the data without the random-effects, and $5.82 \%$ when they were

390 included $\left(R_{m}^{2}=0.00006 ; R_{c}^{2}=0.0582\right)$.

391 Finally, for landscapes as for flowers there was no difference in response time $(\beta=$

$\left.3920.0128, S E=0.0445, \chi^{2}=0.08, p=.774\right)$. This model accounted for $0.02 \%$ of the variance in the

393 data without the random-effects, and $3.62 \%$ when they were included $\left(R_{m}^{2}=0.0002 ; R_{c}^{2}=\right.$

394 0.0362).

395 In summary, for all categories the speeded discrimination was fast and accurate, and

396 differences between categories small. The only additional information was the finding of easier

397 discrimination between symmetrical and original male faces compared to female faces. This

398 pattern is different from that seen for beauty rating: symmetry affected beauty ratings equally for 
399 male and female stimuli, with a trend for a stronger modulation for female rather than male

400 faces.

401

402 Relationship between Beauty and Salience

403 We analysed to what extent the beauty rating reflected the salience of the symmetry in

404 the images. From our first set of analyses we can already see that the category for which

405 symmetry had the strongest effect on beauty rating was that of abstract patterns, and the

406 symmetry for these images was very high. However, symmetry salience was high also for

407 landscapes and here this factor contributed to rating the images as less beautiful. This suggests

408 that symmetry can be used in different ways in different contexts.

409 Here we ask a different question. Within each category, were images rated as more

410 symmetrical also the images rated as more beautiful in the same category? Therefore, we used

411 salience rating as a predictor. We analysed symmetry (original images and images with perfect

412 bilateral symmetry) as a factor. Note that in the first case, symmetry varied among the original

413 objects, such as faces, and in the second case there was perfect bilateral symmetry, but people

414 reported subjective differences in perceived symmetry.

415 Unlike previous analyses here all the categories are entered in the same analysis. To

416 compare them we used a set of pre-planned contrasts. In particular we analysed the difference

417 between landscapes and flowers in one contrast, in another we combined these two to form a

418 new Nature category and compare this to the category of faces. Finally, in another contrast we

419 tested the group of novel stimuli (smooth and angular abstract stimuli) against the familiar

420 objects (faces, flowers and landscapes). The relationship between beauty and salience ratings is

421 represented graphically in scatterplots in Figure 6. 
423 factors. The model revealed that there was an overall effect of salience in predicting preference (

$\left.424 \beta=0.1312, S E=0.0383, \chi^{2}=5.68, p=.017\right)$. Other main effects not related to salience were as

425 follows: Beauty ratings were higher for landscapes than flowers $\left(\beta=-0.448, S E=0.0648, \chi^{2}=\right.$

$426295.48, p<.001)$, for nature (landscapes/flowers) than faces $\left(\beta=-0.4584, S E=0.0389, \chi^{2}=\right.$

$427617.4, p<.001$ ), and for real-world stimuli (landscapes/flowers/faces) than abstract shapes $(\beta=$ -

$\left.4280.2438, S E=0.0221, \chi^{2}=549.77, p<.001\right)$. Many of these main effects also interacted with

429 symmetry and salience. The difference in beauty ratings between abstract stimuli and the other

430 types was smaller for symmetrical than the original stimuli $\left(\beta=-0.0565, S E=0.0222, \chi^{2}=4.3\right.$,

$431 p=.038$ ). Also, increased salience led to a smaller difference in beauty ratings between nature

432 (landscapes/flowers) and faces $\left(\beta=0.0364, S E=0.0403, \chi^{2}=19.99, p<.001\right)$, but a larger

433 difference between landscapes and flowers $\left(\beta=-0.0007, S E=0.0623, \chi^{2}=7.62, p=.006\right)$ and

434 familiar stimuli and abstract shapes $\left(\beta=-0.0149, S E=0.0211, \chi^{2}=9.65, p=.002\right)$.

435 Beauty ratings for familiar shapes (faces, flowers, landscapes) were more strongly

436 affected by symmetry than unfamiliar abstract shapes $\left(\beta=-0.0565, S E=0.0222, \chi^{2}=4.3, p=\right.$

437 .038). Critically, beauty ratings were higher for symmetrical stimuli, but only when this

438 symmetry was salient, as shown by a significant interaction between symmetry and salience $(\beta=$

$\left.439-0.1069, S E=0.032, \chi^{2}=5.39, p=.020\right)$. Finally, there was a three-way interaction between

440 salience, symmetry, and the contrast between nature (landscape/flowers) and face stimuli $(\beta=$

$4410.1015, S E=0.0403, \chi^{2}=5.78, p=.016$ ), which appears to be due to beauty ratings increasing

442 for faces as the salience of their symmetry increases, but increasing or decreasing with salience

443 for the nature items depending on symmetry. This model accounted for $47.22 \%$ of the variance 
444 in the data without the random-effects, and 50.15\% when they were included $\left(R_{m}^{2}=0.4722 ; R_{c}^{2}=\right.$ $4450.5015)$.

446 These effects are hard to visualise. Therefore, in Figure 6 we also present two additional

447 plots. These highlight in particular how symmetry salience could be linked in very different ways

448 to beauty rating depending on which set of stimuli we are analysing: the original or the 449 symmetrical set.

450

\section{Symmetry Preference in Individuals}

452

In the previous section we analysed whether the individual images that were perceived as 453 more symmetrical were also rated as more beautiful. Here we turn to the question of whether, across the stimuli, individuals who liked symmetry did so in a consistent manner. Specifically, we examined whether beauty ratings for symmetrical and original stimuli were consistent across the different categories. For each individual, we computed average beauty response for each category, and separately for the symmetrical and original items. These were different items, but within a category we took the difference between the two averages. Therefore, the new value represents how much more the symmetrical stimuli were rated compared to the original stimuli 460 (per person and per category).

Figure 7 shows a scatterplot matrix in which each dot is a person. The two axes are two categories, and thus people in the top right quadrants liked symmetry in both categories (say,

463 they liked both symmetrical faces and symmetrical landscapes). People in the bottom left

464 quadrants disliked symmetry in both categories, and in the other two quadrants we have 465 inconsistent liking responses. 
467 and the figure show weak consistency in what individuals liked. In many cases they liked

468 symmetry for one category but not for another. Some of these is not surprising, for example

469 comparing abstract shapes and landscapes many participants are in the top left quadrant because

470 they disliked symmetry in landscapes and liked it in abstract shapes. Some other cases are more

471 surprising, like the total absence of consistency for symmetry in faces and in flowers. Here liking

472 symmetry in one category had no relationship with liking symmetry in the other.

473

In the Supplementary materials we also report a second smaller study $(\mathrm{N}=25)$. The

474

procedure and the aims were the same but there were two important changes. One was that in

this study observers saw both the original and the bilateral symmetry versions of the items. The other change was that we included colour version of the images in addition to the greyscale versions. The results were consistent with the results of our main study, thus supporting the generality and robustness of the findings.

\section{Conclusions}

481

Human preference for symmetry and regularity is a well-known phenomenon. In this

study we used a simple rating task with images that were manipulated to introduce perfect

bilateral symmetry around the vertical axis. Vertical bilateral symmetry is the most salient type

of symmetry (Royer, 1981), and is a non-accidental propriety associated with objectness

(Bertamini, 2010; Bertamini, Friedenberg, \& Kubovy, 1997). Human faces are a special example

of bilateral symmetry and attractiveness ratings increase as symmetry increases (Rhodes et al.

images of landscapes which are not characterised by bilateral symmetry in nature. 
490 the symmetrical manipulation (perfect bilateral symmetry). Each observer only saw one item

491 from a given pair. We refer to the two as symmetrical and original, however, original stimuli did

492 possess some degree of symmetry, as is the case for human faces. Therefore, here by

493 symmetrical stimuli we mean stimuli with perfect bilateral symmetry.

We asked what the effect of mixing very different categories of objects would be, and how symmetry preference would relate to symmetry salience in the context of these different categories. We included faces (males and females), abstract shapes (polygons and smoothed polygons), flowers and landscapes. Landscapes are special in that bilateral symmetry here would appear unfamiliar and artificial. The rating for beauty was followed by a second task in which participants rated the salience of symmetry. Finally, they performed a speeded task in which they had to discriminate which of the images in a pair was more symmetrical.

From the ratings of beauty, we can confirm some expected effects. Observers preferred 502 the more symmetrical abstract objects. Independently of symmetry, observers preferred smooth 503 shapes over angular shapes, confirming previous findings for preference for abstract shapes

504 (Bertamini et al., 2016). Probably because within abstract shapes the angularity was a salient

505 factor, this effect was strong. Moreover, observers preferred the more symmetrical faces, in line 506 with previous results (e.g. Little \& Jones 2003; Rhodes et al. 1998). This preference however did 507 not apply to flowers. This may appear inconsistent with what reported by Hůla and Flegr (2016).

508 Note, however, that Hůla and Flegr had found a particular preference for flowers with naturally

509 occurring radial symmetry. The symmetry we introduced was an artificial bilateral reflection.

510 Moreover, Hůla and Flegr only used images of actual flowers while we compared original and 511 modified versions of the flowers. 
512 For landscapes the effect of symmetry reversed compared to faces and abstract shapes,

513 and there was a preference for the original landscape over the one with bilateral symmetry. This

514 suggests that context and categorical classification of images are important. There is no doubt

515 that the landscape images had aesthetic value. Most participants rated landscapes as beautiful

516 (compared to the other categories) as shown in Figure 3. It is likely that introducing perfect

517 symmetry in a natural context was perceived as artificial and unnatural, and consequentially it

518 was disliked. This effect may be similar to that observed for computer generated faces (Zaidel \&

519 Deblieck, 2007).

520 The fact that perfect (artificial) symmetry was not preferred in flowers and disliked in

521 landscapes, suggests that symmetry in the image is not a sufficient factor per se to elicit

522 preference, especially in the natural environment. In the case of flowers, the study by Hůla \&

523 Flegr (2016) found that beauty was related to radial symmetry. They also had ratings of

524 prototypically and therefore they could show that what was judged beautiful was related to what 525 was perceived as the more prototypical image of a flower. For a prototypical wildflower radial

526 rather that bilateral symmetry is the most important factor. In the case of landscapes this was the

527 only category in which symmetry was not associated with an object but rather with a layout of

528 features and objects. In other words, for landscapes symmetry is about composition. However,

529 despite the fact that for landscapes there was a tendency to dislike symmetry, it is remarkable

530 that within the set of symmetrical images of landscapes the higher ratings of beauty were given

531 to those rated as more symmetrical. We consider this surprising result when discussing the

532 correlation between salience and beauty.

533 Natural landscapes are unlikely to be symmetrical, but often humans manipulate the

534 landscape and introduce regularities. This is clearly the case for landscaped gardens in particular 
535 starting with the Italian Renaissance style (Leon Battista Alberti, 1404-1472, wrote a book about

536 architecture that talks extensively about gardens). A recent study, however, has found that the

537 restorative power of gardens was higher for informal rather than formal gardens (Twedt, Rainey, 538 \& Proffitt, 2016).

539 In a second analysis we tested ratings of symmetry salience as a predictor of beauty

540 ratings. Overall, we confirmed this link, as it was already observed for faces in the original paper

541 by Rhodes et al. (1998). However, the comparison of the different categories revealed a complex

542 pattern (Figure 6). While previous work has already established a positive relationship between

543 symmetry salience and symmetry preference for abstract patterns (Makin et al., 2012), this did

544 not generalize to landscapes.

545 The experiment used images in greyscale, and a design that avoided presenting both

546 original and modified versions of an item to a given observer. Colour is likely to contribute to

547 beauty. In a follow-up study we included some of the categories (abstract, flowers, landscapes)

548 in both colour and greyscale. We report this experiment in the supplementary materials. It

549 confirmed that ratings for beauty were higher in the case of colour images, but it also confirmed

550 and supported the other results without interactions with colour (see Figure 1S).

551 In terms of general preference for symmetry in some individuals and not others we did

552 not find much evidence that strong symmetry preference in one category implied strong

553 preference in another category. Individual differences have recently been studied in the context

554 of visual illusions. Grzeczkowski et al. (2017) asked the question of common factors in a sample

555 of over one hundred participants. If these common factors exist, a person susceptible to one

556 illusion should be susceptible to other illusions as well. They looked at the correlations between

557 the strength of six illusions but could only confirm a correlation between two of them 
558 (Ebbinghaus and Ponzo). They came to the conclusion that, in terms of visual perception,

559 individuals are unique.

560 We highlight two novel aspects of the results. First the role of symmetry varies between

561 categories, as we have seen, and landscape with bilateral symmetry were liked less than the

562 original images of landscapes. We argue that bilateral symmetry is associated with objects and

563 not images with a layout of multiple objects (trees, mountains etc.). However, this first

564 consideration has to be interpreted keeping in mind a second aspect of the data. Within the

565 original images salience and beauty ratings were negatively correlated. However, within the new

566 symmetrical images of landscapes there was a preference for the more symmetrical images

567 (more precisely those where symmetry was more salient, and ratings of subjective symmetry

568 higher). Although we do not have self-reports on this, it is probable that observers were not

569 aware of this effect of symmetry on their responses, as all images were presented interleaved in a

570 random order. We suggest that symmetry was not related to beauty for landscapes because

571 variation in the layout is a positive quality of a complex landscape. But symmetry was related to

572 beauty for artificial landscapes because these were treated as if they were patterns, or objects,

573 and therefore more similar to the other categories of stimuli.

574 The context effects that we report are further evidence of how difficult it is to attribute

575 preference to specific factors. Philosophers have expressed scepticism (e.g., Dickie, 1962), but

576 even within empirical aesthetics problems have been noted (Holmes \& Zanker, 2012), and one

577 problem is particular is known as the Gestalt nightmare: having a preference for symmetry and

578 for blue does not mean that we can sum these preferences to predict responses to blue

579 symmetrical stimuli (Makin, 2017). Although strong claims have been made about the existence

580 of a "single neural currency" for aesthetics (Skov \& Nadal, 2018), it would be a mistake to 
581 conclude from the existence of common reward mechanisms that fixed, objective factors are

582 monotonically contributing to reward, or to preference. Our observed context effects are

583 consistent with other context effects reported. For example, for faces there are both assimilation

584 and contrast effects (Wedell, Parducci, \& Geiselman, 1987). More generally, the nature of

585 human choices is known to be multi-dimensional (Tversky \& Shafir, 1992). In evaluating visual

586 preference, we found that symmetry can play both a positive and a negative role even within the

587 same study and for the same category of objects. The critical factor that can reverse the effect of

588 symmetry is its association with an object: bilateral symmetry is expected to be a within-object

589 property and is only judged as beautiful in that context. 
591 Albrecht, S., \& Carbon, C.-C. (2014). The Fluency Amplification Model: Fluent stimuli show 592 more intense but not evidently more positive evaluations. Acta Psychologica, 148, 195593 203. https://doi.org/10/f5xcr6

594 Barr, D. J., Levy, R., Scheepers, C., \& Tily, H. J. (2013). Random Effects Structure for 595 Confirmatory Hypothesis Testing: Keep It Maximal. Journal of Memory and Language, 68(3), 255-278. https://doi.org/10/gcm4wc

Bates, D., Mächler, M., Bolker, B., \& Walker, S. (2015). Fitting Linear Mixed-Effects Models 598 Using Lme4. Journal of Statistical Software, 67(1). https://doi.org/10/gcrnkw

Bertamini, M., Friedenberg, J. D., \& Kubovy, M. (1997). Detection of symmetry and perceptual 600 organization: The way a lock-and-key process works. Acta Psychologica, 95(2), 119

601 140. https://doi.org/10/cctvqj

602 603

604 605

606

607

608 609

610

611
Royer, F. L. (1981). Detection of Symmetry. Journal of Experimental Psychology: Human Perception \& Performance, 7, 1186-1210. https://doi.org/10/bvmvth

Bertamini, M. (2010). Sensitivity to Reflection and Translation Is Modulated by Objectness. Perception, 39(1), 27-40. https://doi.org/10/cnv89m

Bertamini, M., Palumbo, L., Gheorghes, T. N., \& Galatsidas, M. (2016). Do Observers like Curvature or Do They Dislike Angularity? British Journal of Psychology, 107(1), 154178. https://doi.org/10/gd84vw

Birkhoff, G. D. (1933). Aesthetic Measure (Vol. 38). Cambridge: Harvard University Press. Chatterjee, A. (2014). The aesthetic brain: How we evolved to desire beauty and enjoy art. New York, NY: Oxford University Press. 
612 Che, J., Sun, X., Gallardo, V., \& Nadal, M. (2018). Chapter 5 - Cross-cultural empirical

613 aesthetics. In J. F. Christensen \& A. Gomila (Eds.), Progress in Brain Research (Vol. 237, 614 pp. 77-103). Elsevier. https://doi.org/10.1016/bs.pbr.2018.03.002

615 Dickie, G. (1962). Is Psychology Relevant to Aesthetics? The Philosophical Review, 71(3), 285616 302. https://doi.org/10/fm6vxx

617 Eisenman, R. (1967). Complexity-Simplicity: I. Preference for Symmetry and Rejection of 618 Complexity. Psychonomic Science, 8(4), 169-170. https://doi.org/10/gd84v3

619 Enquist, M., \& Arak, A. (1994). Symmetry, Beauty and Evolution. Nature, 372(6502), 169-172. 620 https://doi.org/10/d28q4g

621 Enquist, M., \& Johnstone, R. A. (1997). Generalization and the Evolution of Symmetry 622 Preferences. Proceedings of the Royal Society of London B: Biological Sciences, 623 264(1386), 1345-1348. https://doi.org/10/bg9xvk

624 Eysenck, H. J., \& Castle, M. (1970). TRAINING IN ART AS A FACTOR IN THE 625 DETERMINATION OF PREFERENCE JUDGEMENTS FOR POLYGONS. British Journal of Psychology, 61(1), 65-81. https://doi.org/10/bpzxkn

Falk, J. H., \& Balling, J. D. (2010). Evolutionary Influence on Human Landscape Preference. Environment and Behavior, 42(4), 479-493. https://doi.org/10/cr5vsd

629 Gangestad, S. W., \& Thornhill, R. (1997). The Evolutionary Psychology of Extrapair Sex: The 630 Role of Fluctuating Asymmetry. Evolution and Human Behavior, 18(2), 69-88.

631 https://doi.org/10/dvmd9q

632 Ghirlanda, S., Jansson, L., \& Enquist, M. (2002). Chickens prefer beautiful humans. Human 633 nature, 13(3), 383-389. 
634 Grzeczkowski, L., Clarke, A. M., Francis, G., Mast, F. W., \& Herzog, M. H. (2017). About 635 individual differences in vision. Vision Research, 141, 282-292.

636 https://doi.org/10/gcszm6

637 Höfel, L., \& Jacobsen, T. (2007). Electrophysiological Indices of Processing Symmetry and 638 Aesthetics: A Result of Judgment Categorization or Judgment Report? Journal of Psychophysiology, 21(1), 9. https://doi.org/10/fdcjrn

Holmes, T., \& Zanker, J. M. (2012). Using an Oculomotor Signature as an Indicator of Aesthetic 641 Preference. I-Perception, 3(7), 426-439. https://doi.org/10/gcdtjp

642 643

644 645

646 647 648

651

652

653

654
Hůla, M., \& Flegr, J. (2016). What Flowers Do We like? The Influence of Shape and Color on the Rating of Flower Beauty. PeerJ, 4, e2106. https://doi.org/10/gd84v4

Humphrey, D. (1997). Preferences in Symmetries and Symmetries in Drawings: Asymmetries between Ages and Sexes. Empirical Studies of the Arts, 15(1), 41-60. https://doi.org/10/fq6jhz

Jacobsen, T., \& Höfel, L. (2003). Descriptive and evaluative judgment processes: behavioral and electrophysiological indices of processing symmetry and aesthetics. Cognitive, Affective, \& Behavioral Neuroscience, 3(4), 289-299. https://doi.org/10/dnmbh9

Johnson, P. C. D. (2014). Extension of Nakagawa \& Schielzeth's $R^{2}{ }_{\text {GLMM }}$ to Random Slopes Models. Methods in Ecology and Evolution, 5(9), 944-946. https://doi.org/10/f6j4dj

Leder, H., Belke, B., Oeberst, A., \& Augustin, D. (2004). A Model of Aesthetic Appreciation and Aesthetic Judgments. British Journal of Psychology, 95(4), 489-508. https://doi.org/10/cs7p65 
655 Little, A. C. (2014). Domain Specificity in Human Symmetry Preferences: Symmetry Is Most 656 Pleasant When Looking at Human Faces. Symmetry, 6(2), 222-233.

657 https://doi.org/10/gcfpxt

658 Little, A. C., \& Jones, B. C. (2003). Evidence against Perceptual Bias Views for Symmetry 659 Preferences in Human Faces. Proceedings of the Royal Society of London B: Biological

660 Sciences, 270(1526), 1759-1763. https://doi.org/10/dmv2xv

661

662

Makin, A. D. J. (2017). The Gap between Aesthetic Science and Aesthetic Experience. Journal

663

664

665

666

667

668

669

670

671

672

673

674

675 of Consciousness Studies, 24(1-2), 184-213.

Makin, A. D. J., Helmy, M., \& Bertamini, M. (2018). Visual cortex activation predicts visual preference: Evidence from Britain and Egypt. Quarterly Journal of Experimental Psychology, 71(8), 1771-1780. https://doi.org/10/gfr94m

Makin, A. D. J., Pecchinenda, A., \& Bertamini, M. (2012). Grouping by closure influences subjective regularity and implicit preference. I-Perception, 3(8), 519-527. https://doi.org/10/gcdwh6

Makin, A. D. J., Pecchinenda, A., \& Bertamini, M. (2012). Implicit Affective Evaluation of Visual Symmetry. Emotion, 12(5), 1021-1030. https://doi.org/10/fzck29

Makin, A. D. J., Wright, D., Rampone, G., Palumbo, L., Guest, M., Sheehan, R., ... Bertamini, M. (2016). An electrophysiological index of perceptual goodness. Cerebral Cortex, 26(12). https://doi.org/10/f9nmvd

McManus, I. C. (2005). Symmetry and asymmetry in aesthetics and the arts. European Review, 13, 157-180. https://doi.org/10/fr2v99 
676 Nakagawa, S., \& Schielzeth, H. (2013). A General and Simple Method for Obtaining $R^{2}$ from Generalized Linear Mixed-Effects Models. Methods in Ecology and Evolution, 4(2),

678 133-142. https://doi.org/10/f4pkjx

679 680 681 682 683 684 685 686 687 688 689 690 691 692 693 694 695 696 697

Nakagawa, S., Johnson, P. C. D., \& Schielzeth, H. (2017). The Coefficient of Determination $R^{2}$ and Intra-Class Correlation Coefficient from Generalized Linear Mixed-Effects Models Revisited and Expanded. Journal of The Royal Society Interface, 14(134), 20170213. https://doi.org/10/gddpnq

Oliva, A., \& Schyns, P. G. (2000). Diagnostic Colors Mediate Scene Recognition. Cognitive Psychology, 41(2), 176-210. https://doi.org/10/cfs6cf

Oliva, A., \& Torralba, A. (2001). Modeling the Shape of the Scene: A Holistic Representation of the Spatial Envelope. International Journal of Computer Vision, 42(3), 145-175. https://doi.org/10/dm4d3b

Orians, G. H., \& Heerwagen, J. H. (1992). Evolved Responses to Landscapes. In J. H. Barkow, L. Cosmides, \& J. Tooby (Eds.), The Adapted Mind: Evolutionary Psychology and the Generation of Culture (pp. 555-579). New York: Oxford University Press.

Osborne, H. (1986). Symmetry as an aesthetic factor. Computers \& Mathematics with Applications, 12(1-2), 77-82. https://doi.org/10/dnvfx9

Palumbo, L., Ruta, N., \& Bertamini, M. (2015). Comparing Angular and Curved Shapes in Terms of Implicit Associations and Approach/Avoidance Responses. PLOS ONE, 10(10), e0140043. https://doi.org/10/gd84v6

Peirce, J. W. (2007). PsychoPy: Psychophysics Software in Python. Journal of Neuroscience Methods, 162(1), 8-13. https://doi.org/10/b5dqq8 
698 Perrett, D. I., May, K. A., \& Yoshikawa, S. (1994). Facial Shape and Judgements of Female

699 Attractiveness. Nature, 368(6468), 239-242. https://doi.org/10/dbv33k

700 Pound, N., Lawson, D. W., Toma, A. M., Richmond, S., Zhurov, A. I., \& Penton-Voak, I. S.

701 (2014). Facial fluctuating asymmetry is not associated with childhood ill-health in a large

702 British cohort study. Proceedings of the Royal Society B: Biological Sciences, 281(1792),

20141639-20141639. https://doi.org/10/gft84n

704 R Core Team. (2018). R: A Language and Environment for Statistical Computing. Vienna,

$705 \quad$ Austria: R Foundation for Statistical Computing.

706 Ramachandran, V. S., \& Hirstein, W. (1999). The Science of Art: A Neurological Theory of

$707 \quad$ Aesthetic Experience. Journal of Consciousness Studies, 6(6-7), 15-51.

708 Reber, R., Schwarz, N., \& Winkielman, P. (2004). Processing Fluency and Aesthetic Pleasure: Is

709 Beauty in the Perceiver's Processing Experience? Personality and Social Psychology

Review, 8(4), 364-382. https://doi.org/10/c7cq8z

711 Redies, C. (2007). A Universal Model of Esthetic Perception Based on the Sensory Coding of

712 Natural Stimuli. Spatial Vision, 21(1), 97-117. https://doi.org/10/dj6vs9

713 Rhodes, G. (2006). The Evolutionary Psychology of Facial Beauty. Annual Review of

714 Psychology, 57(1), 199-226. https://doi.org/10/ddg2hh

715 Rhodes, G., Proffitt, F., Grady, J. M., \& Sumich, A. (1998). Facial Symmetry and the Perception

716 of Beauty. Psychonomic Bulletin \& Review, 5(4), 659-669. https://doi.org/10/ddzs8x

717 Rodriguez, I., Gumbert, A., de Ibarra, N., Kunze, J., \& Giurfa, M. (2004). Symmetry is in the eye

718 of the beeholder: innate preference for bilateral symmetry in flower-naive bumblebees.

719 Naturwissenschaften, 91(8), 374-377. https://doi.org/10.1007/s00114-004-0537-5 
720 Ryan, M. (2018). A taste for the beautiful: the evolution of attraction. Princeton University

721

722

723

724

725

726

727

728

729

730

731

732

733

734

735

736

737

738

739

740

741 Press.

Skov, M., \& Nadal, M. (2018). Art Is Not Special: An Assault on the Last Lines of Defense against the Naturalization of the Human Mind. Reviews in the Neurosciences, 29(6), 699702. https://doi.org/10/gcwvhk

Tversky, A., \& Shafir, E. (1992). Choice under Conflict: The Dynamics of Deferred Decision. Psychological Science, 3(6), 358-361. https://doi.org/10/dcr3jg

Twedt, E., Rainey, R. M., \& Proffitt, D. R. (2016). Designed Natural Spaces: Informal Gardens Are Perceived to Be More Restorative than Formal Gardens. Frontiers in Psychology, 7. https://doi.org/10/gfc4rw

van der Helm, P. A., \& Leeuwenberg, E. L. J. (1996). Goodness of visual regularities: A nontransformational approach. Psychological Review, 103, 429-456. https://doi.org/10/cvcjmt

Vessel, E. A., Maurer, N., Denker, A. H., \& Starr, G. G. (2018). Stronger Shared Taste for Natural Aesthetic Domains than for Artifacts of Human Culture. Cognition, 179, 121131. https://doi.org/10/gd6z8b

Watson, P. J., \& Thornhill, R. (1994). Fluctuating Asymmetry and Sexual Selection. Trends in Ecology \& Evolution, 9(1), 21-25. https://doi.org/10/fsz6gj

Wedell, D. H., Parducci, A., \& Geiselman, R. E. (1987). A formal analysis of ratings of physical attractiveness: Successive contrast and simultaneous assimilation. Journal of Experimental Social Psychology, 23(3), 230-249. https://doi.org/10/frrd7g

Wilson, E. (1984). Biophilia. Cambridge (MA): Harvard University Press. 
742 Yue, X., Vessel, E. A., \& Biederman, I. (2007). The neural basis of scene preferences.

$743 \quad$ Neuroreport, 18(6), 525-529. https://doi.org/10/d83fpd

744 Zaidel, D. W., \& Hessamian, M. (2010). Asymmetry and Symmetry in the Beauty of Human

$745 \quad$ Faces. Symmetry, 2(1), 136-149. https://doi.org/10/d6p9z2 


\section{Figure 1}

Figure 1

Examples of images from each of the six categories. The top row shows the original images that differed in level of symmetry. The second row shows perfect bilateral symmetry. For faces this was a morph of left and right sides and for all other categories was based on the left side only. The unoccluded faces can be seen in the original paper (Rhodes, Proffitt, Grady, \& Sumich, 1998) here: www.doi.org/10/ddzs8x

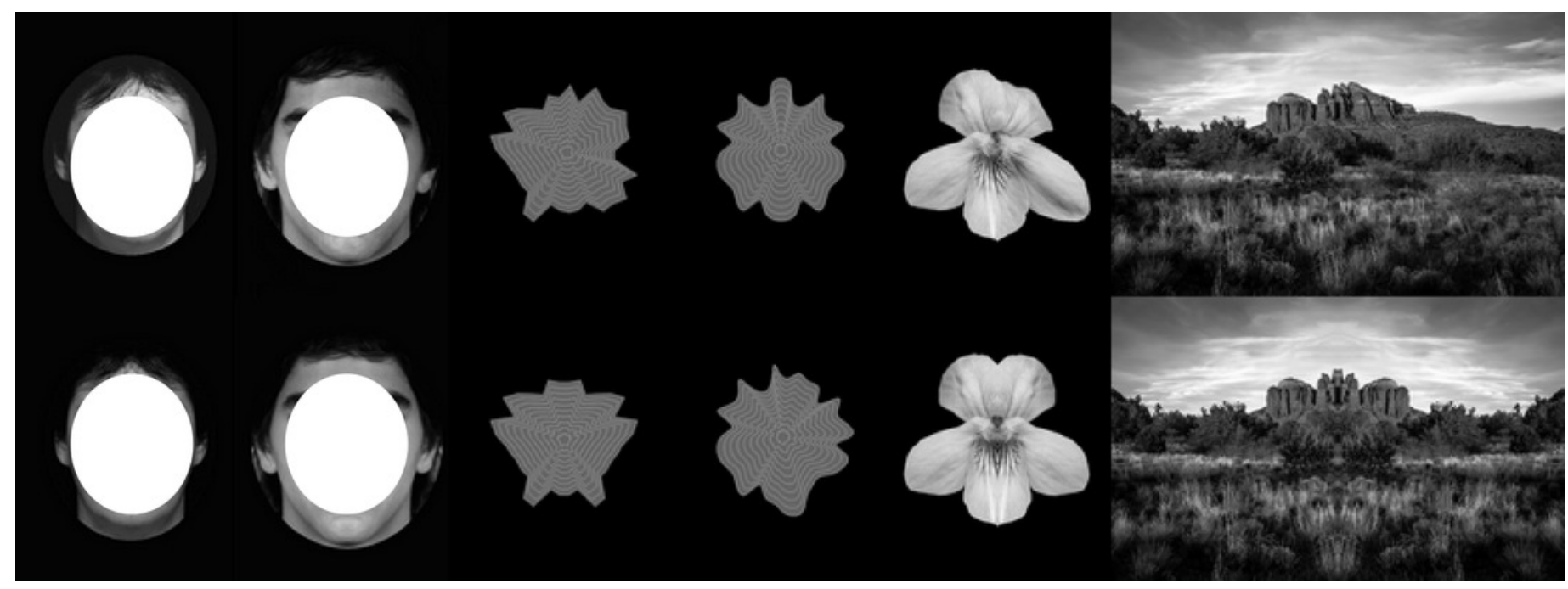




\section{Figure 2}

Figure 2

An illustration of the three tasks: beauty rating, followed by salience rating, followed by speeded discrimination. The stimuli were the same for the three tasks but were presented in different random order. The unoccluded faces can be seen in the original paper (Rhodes, Proffitt, Grady, \& Sumich, 1998) here: www.doi.org/10/ddzs8x 
Welcome to the Experiment.

You will be presented with several images. You will need to indicate how much you like each image (from not at all to very beautiful). First, you will have some practice trials without the actual images to familiarize with the rating scale.

Press space to start with the practice.

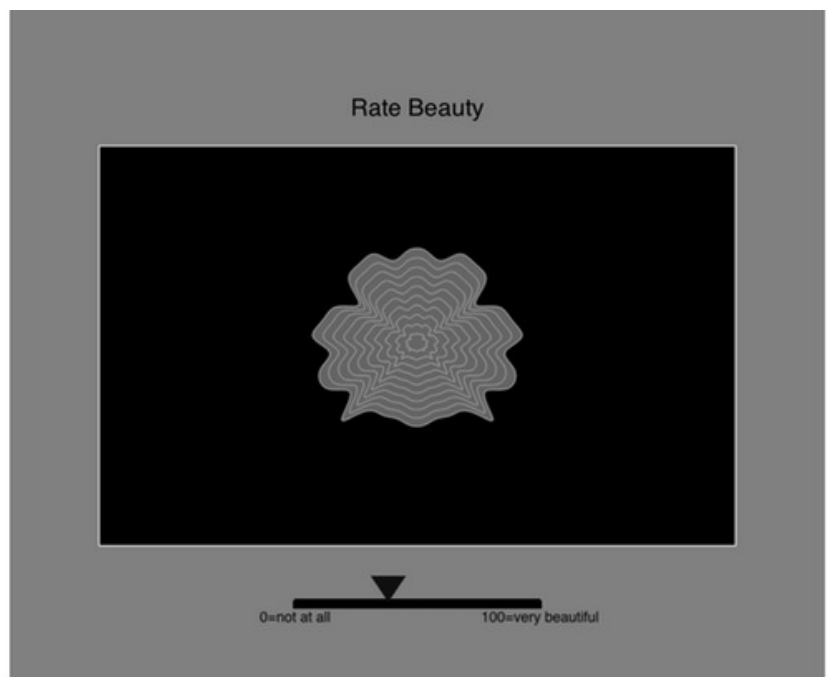

You will be presented with the same images again.

You will need to indicate how

'obvious' or 'salient' is the symmetry

in each image (from not at all at very

salient).

Press space bar to start.'

You will be presented with pairs of images.

You will need to indicate which is more symmetrical (if left press ' $z$ ' if right press ' $m$ ').

For this task you need to go as FAST

as you can without making errors.

Press space bar to start.
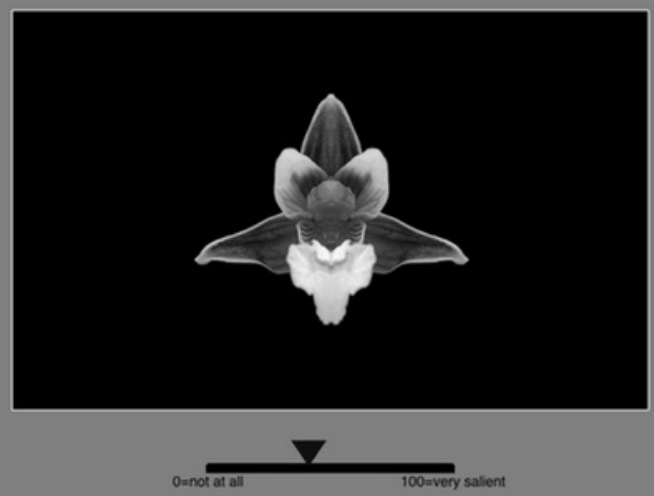

Choose Left or Right

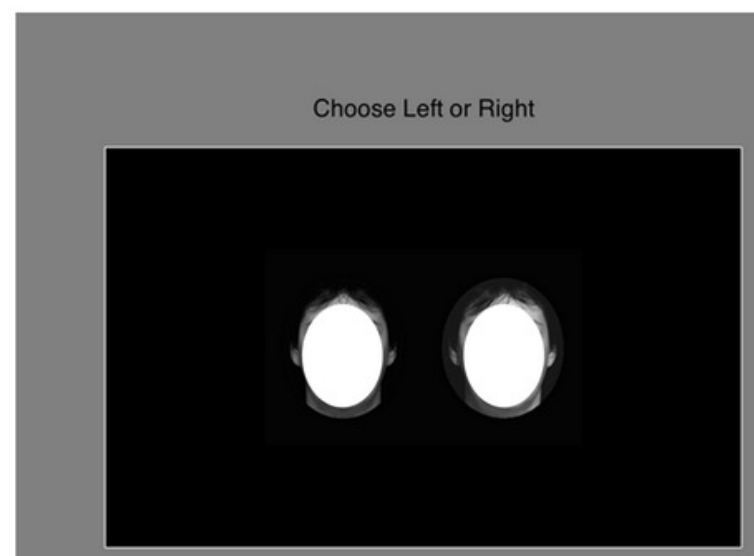

Rate Symmetry 


\section{Figure 3 (on next page)}

\section{Beauty ratings}

Mean rating for the beauty task, comparing original stimuli and stimuli with bilateral symmetry ( $x$ axis). The panels are organised by category and match the way the statistical analyses have been carried out. Error bars represent the standard error adjusted for the model random effects structure. The scale has the same range in the panels but is shifted upwards in the last one. 
(A) Faces Beauty Ratings

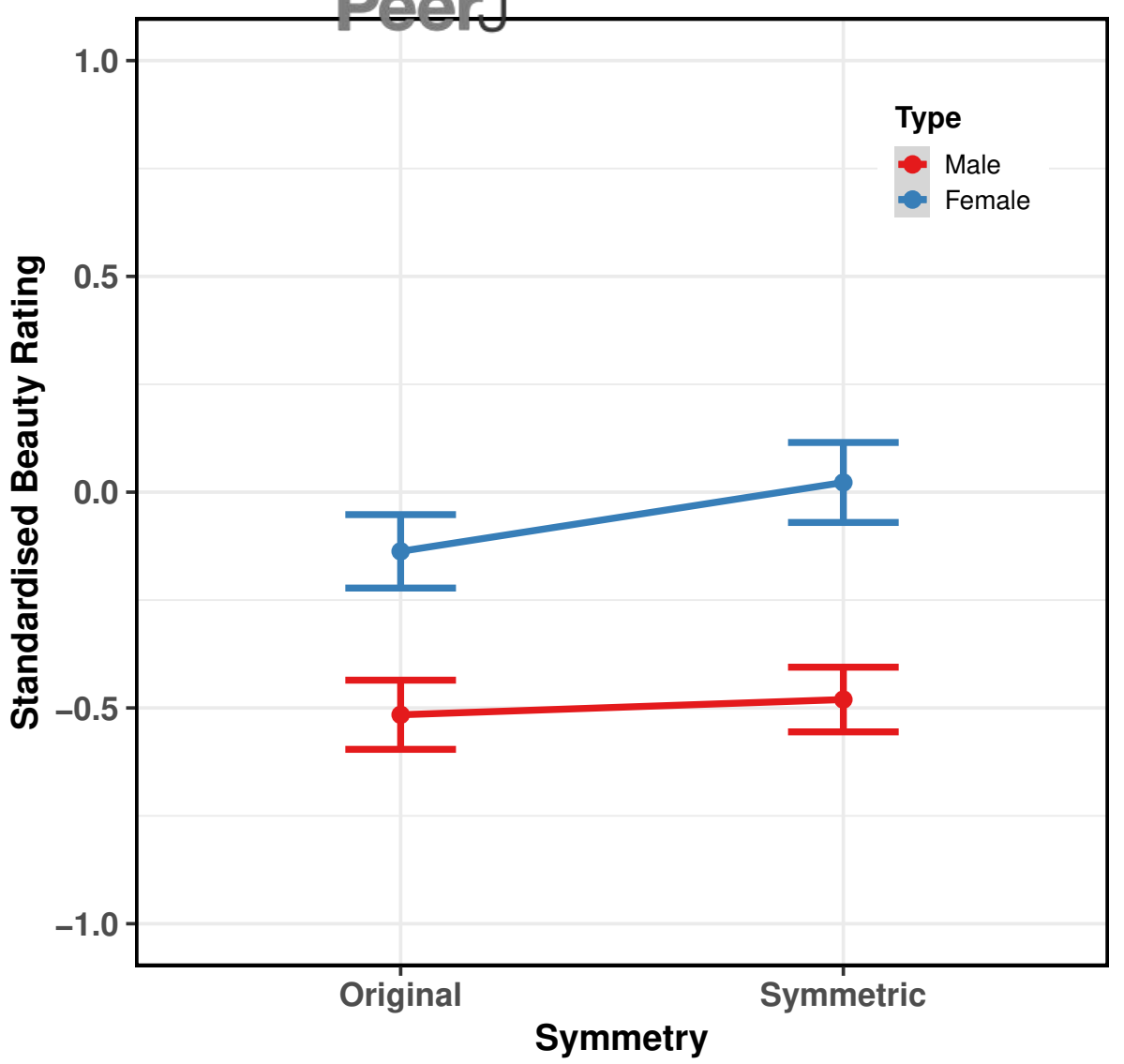

(C) Flower - Beauty Ratings

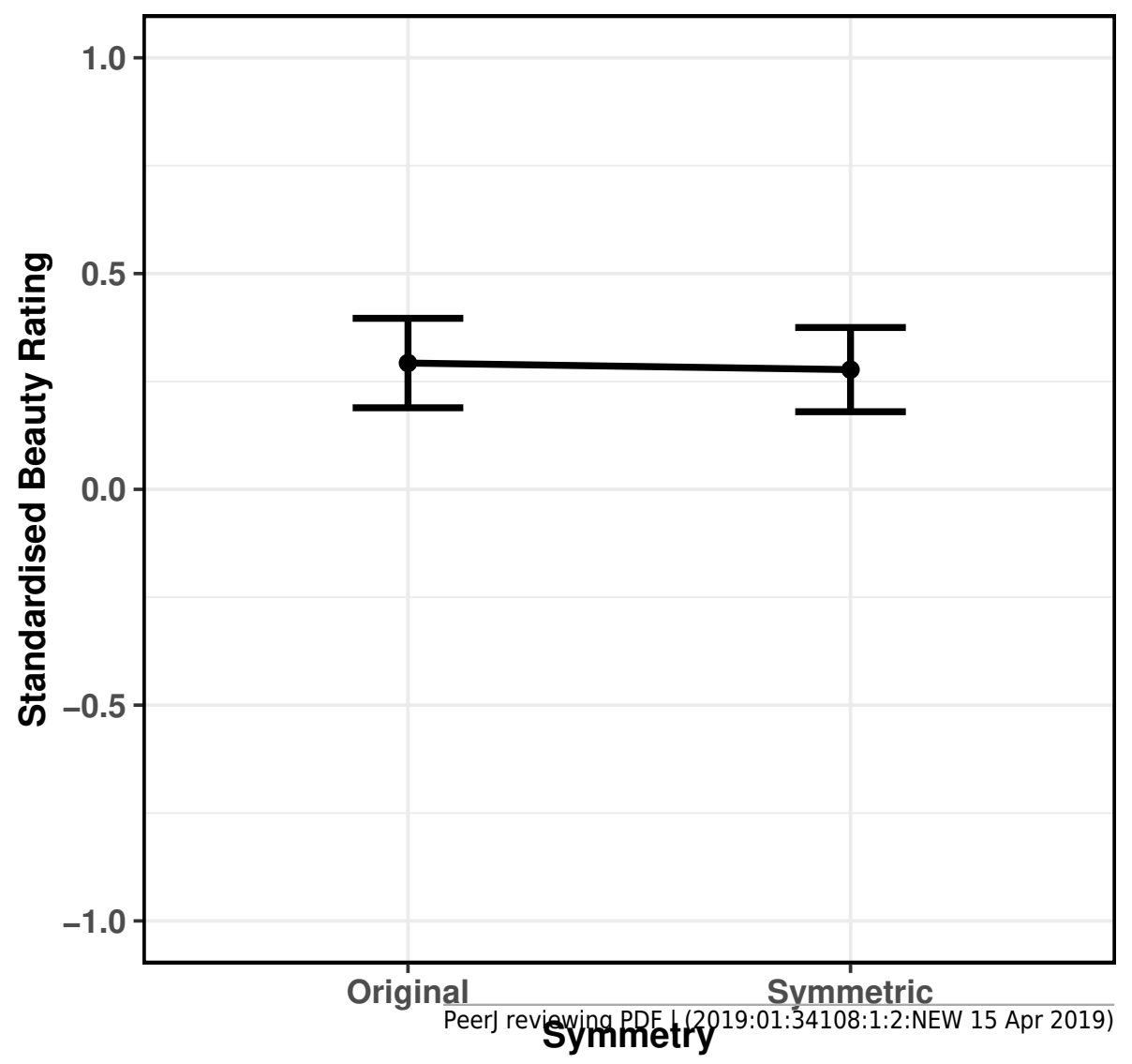

(B) Abstract - Beauty Ratings

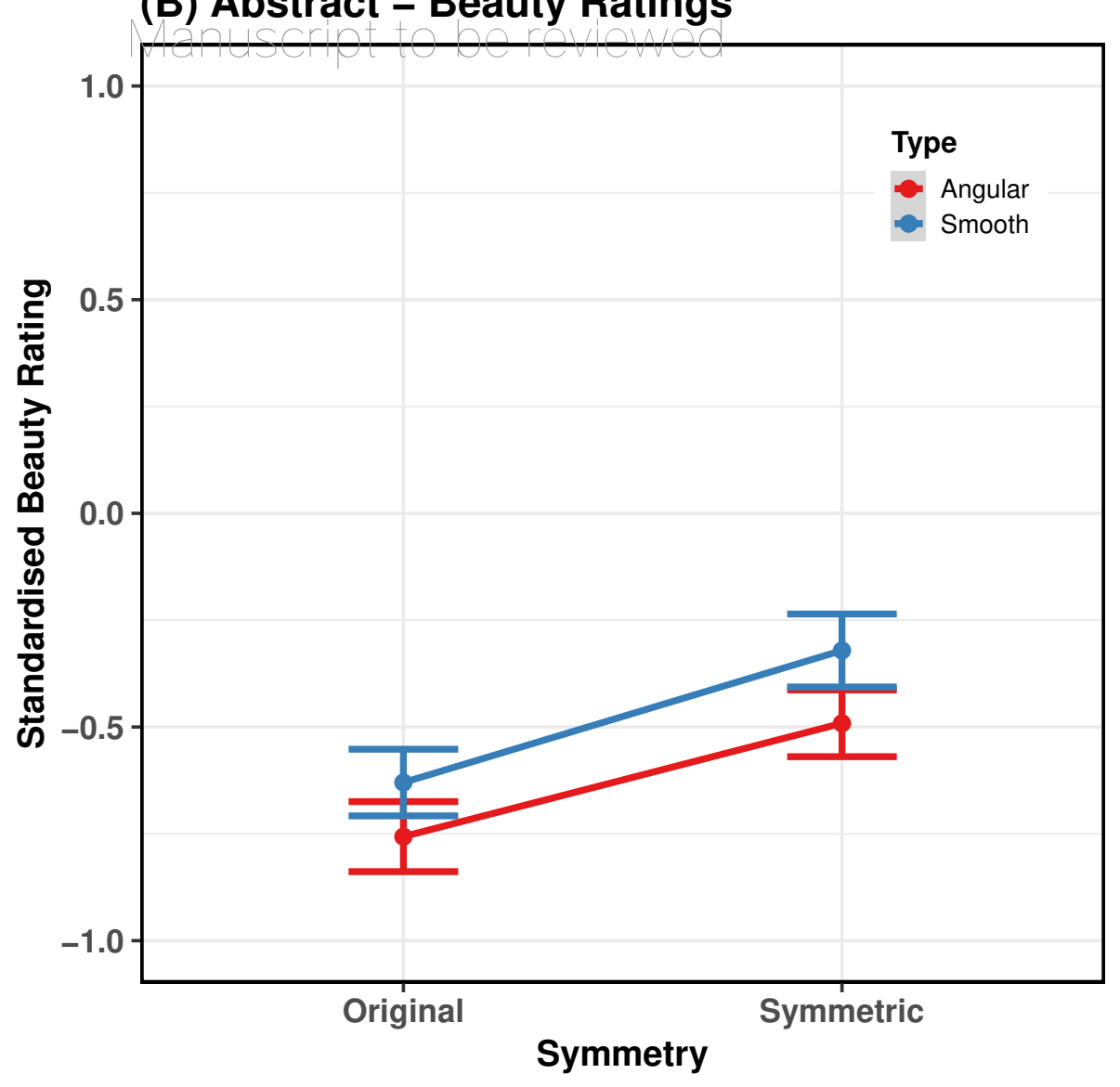

(D) Landscape - Beauty Ratings

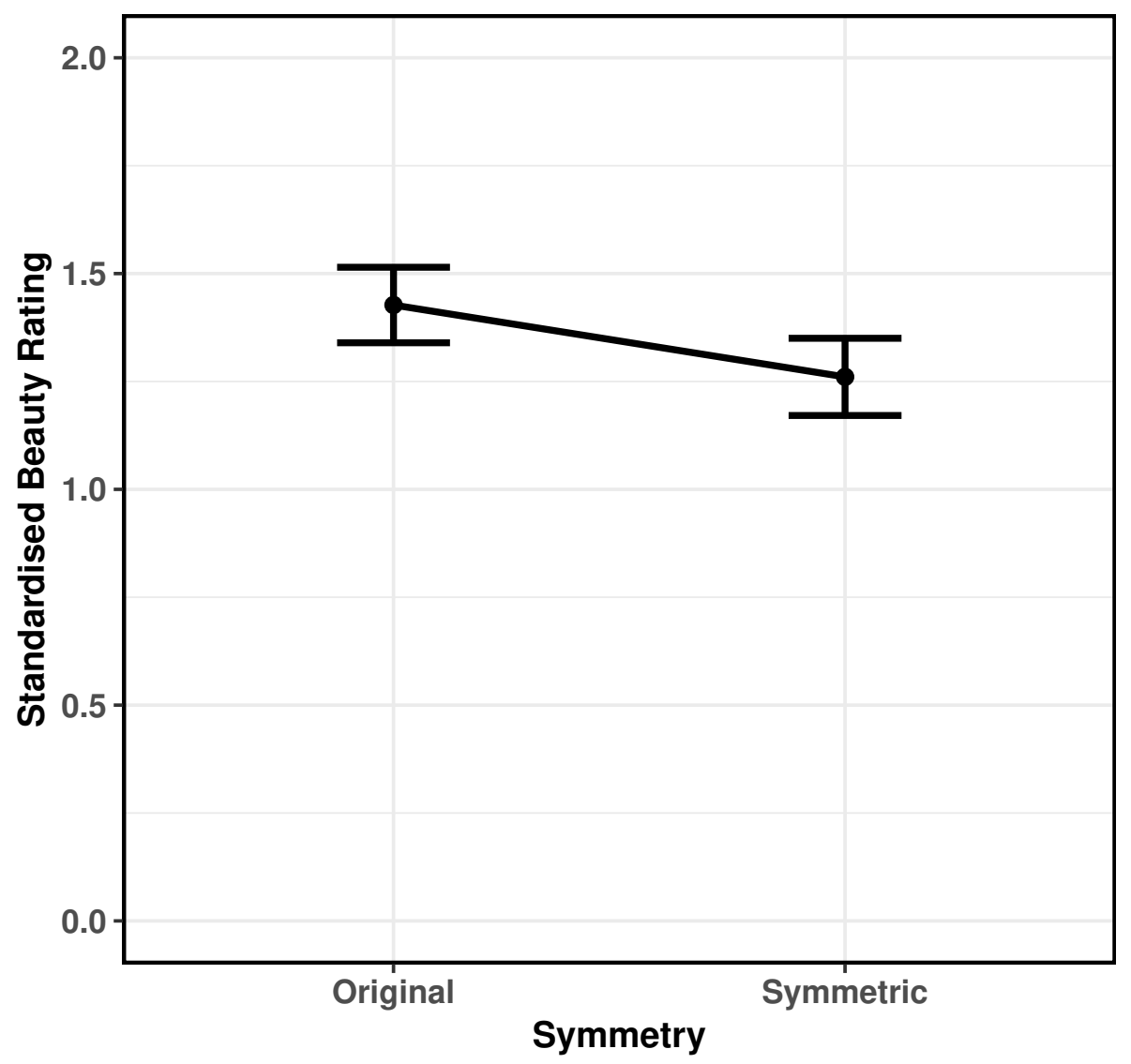


Figure 4 (on next page)

Salience ratings

Mean rating for the symmetry salience task, comparing original stimuli and stimuli with bilateral symmetry ( $x$ axis). The panels are organised by category and match the way the statistical analyses have been carried out. Error bars represent the standard error adjusted for the model random effects structure. 
Figure $\mathbf{5}$ (on next page)

Speeded discrimination time

Mean speeded discrimination task, comparing original stimuli and stimuli with bilateral symmetry ( $x$ axis). The panels are organised by category and match the way the statistical analyses have been carried out. Error bars represent the standard error adjusted for the model random effects structure. 
(A) Faces - Response Times

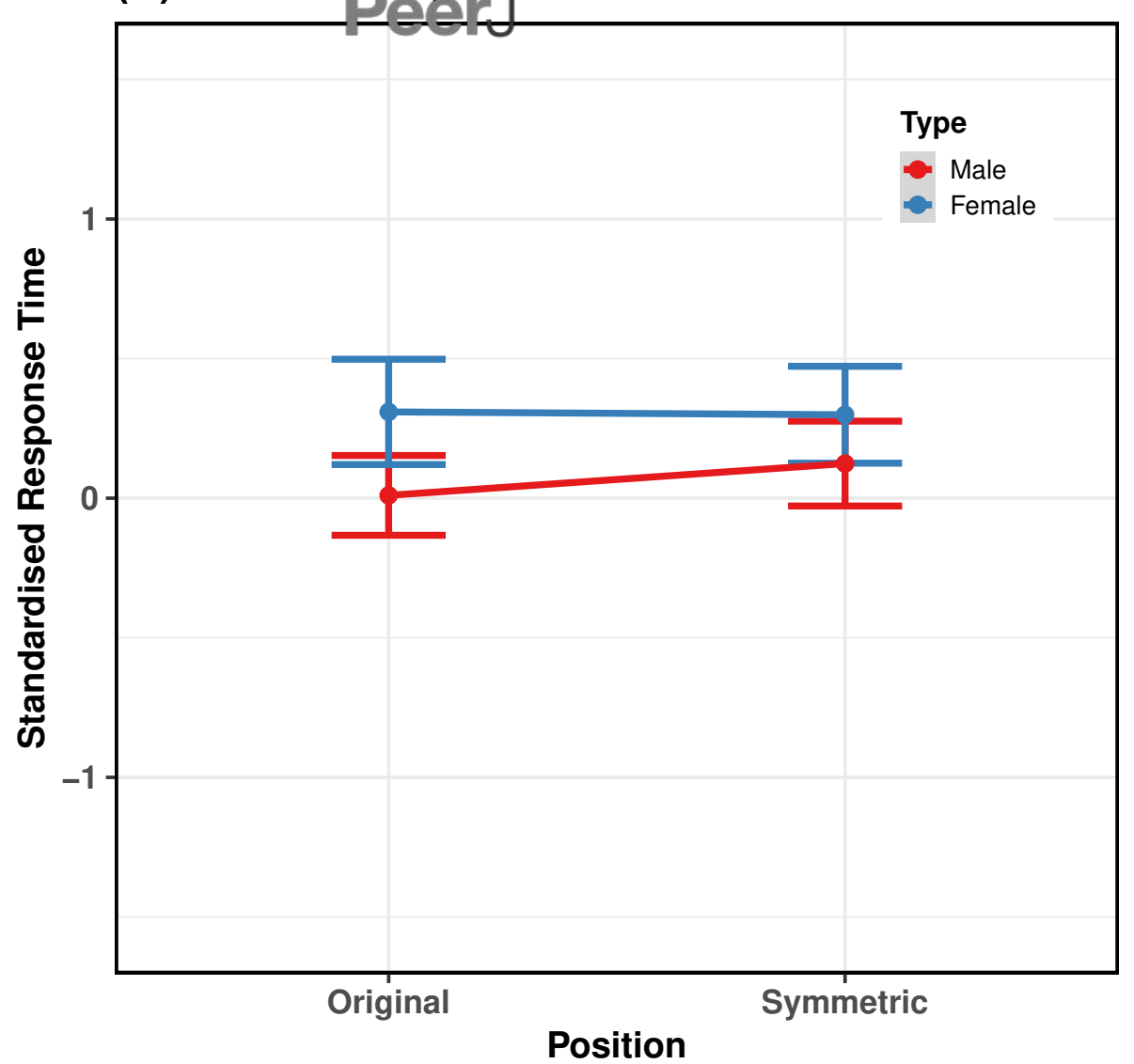

(C) Flower - Response Times

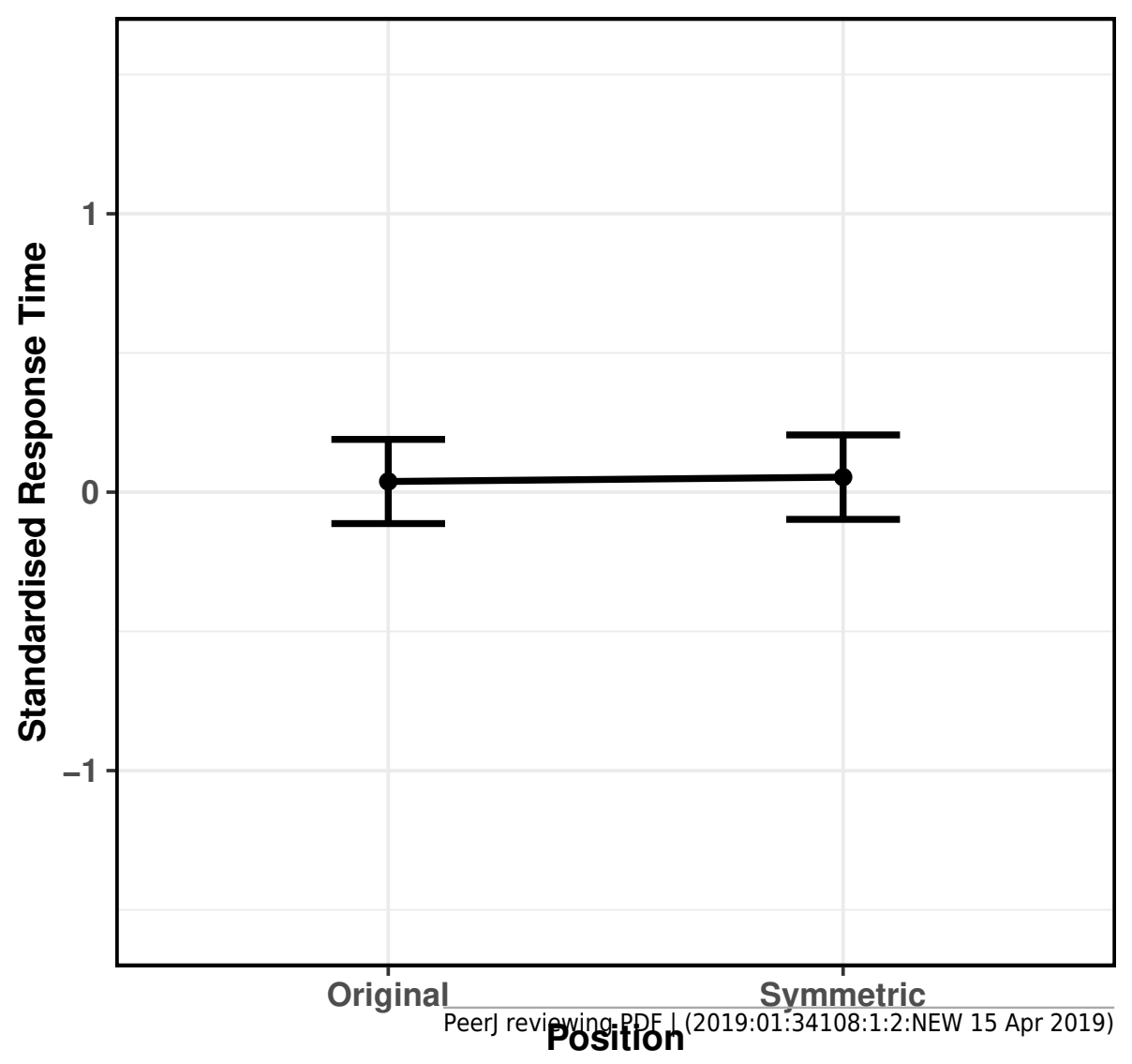

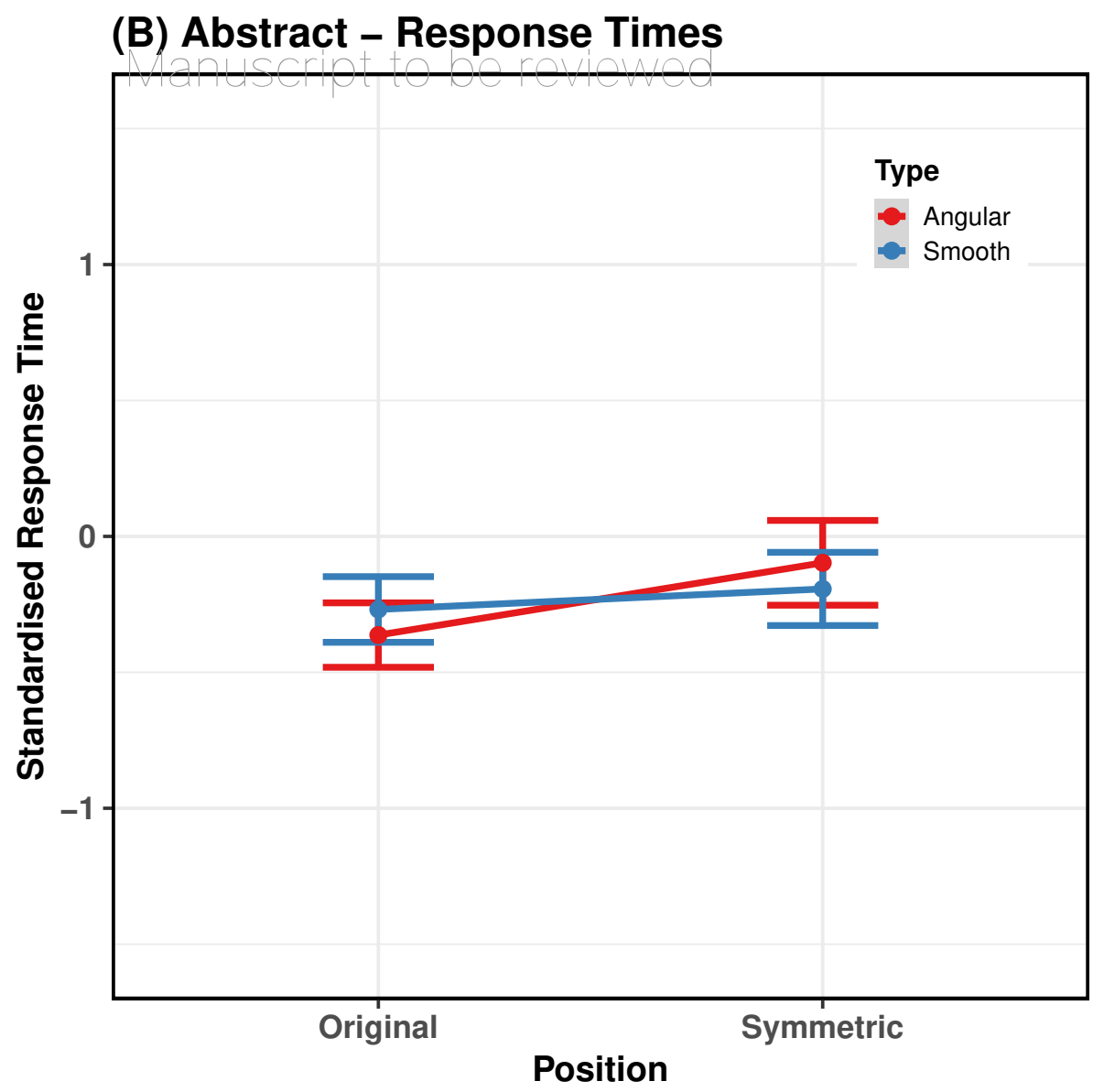

(D) Landscape - Response Times

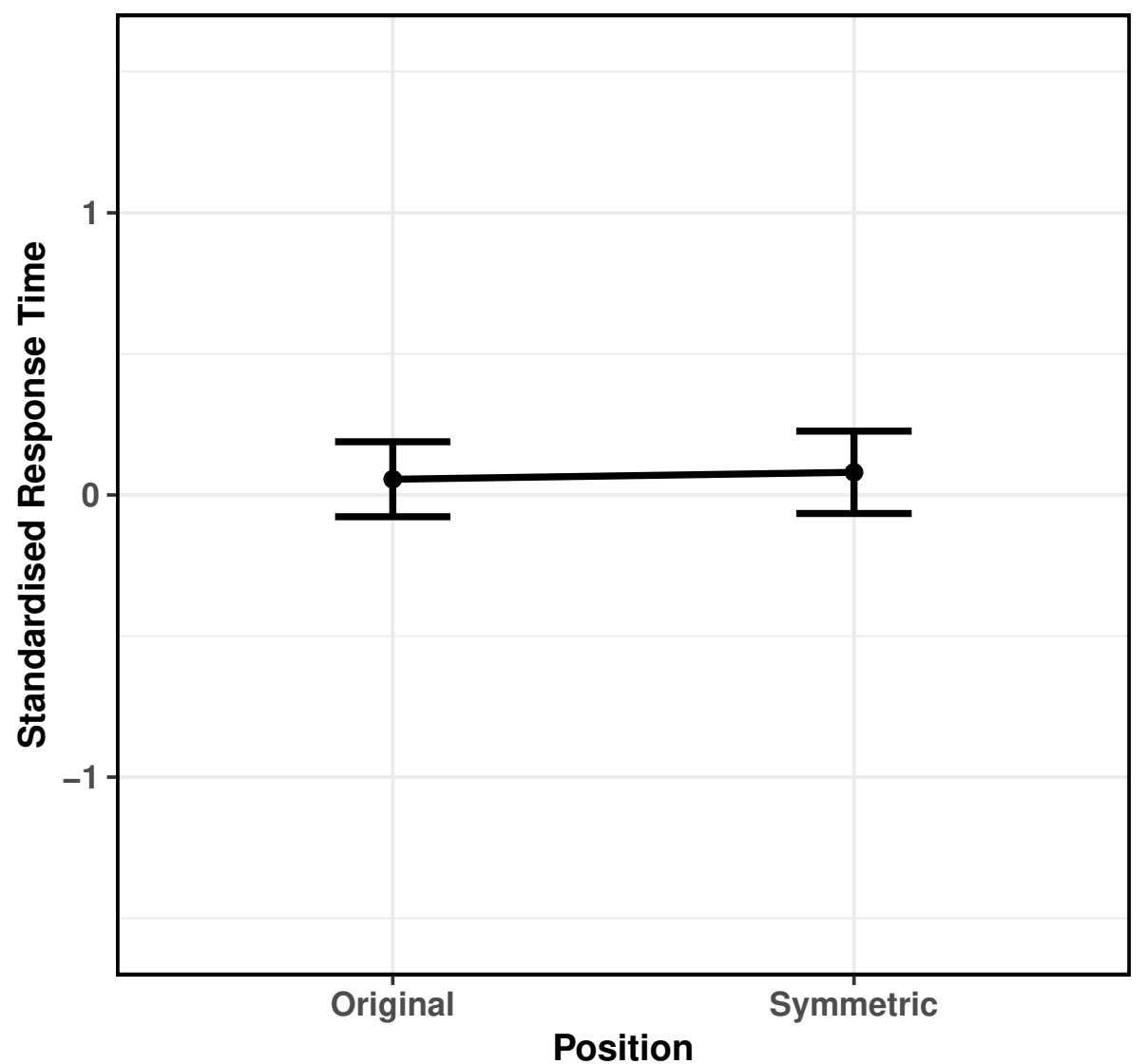




\section{Figure 6 (on next page)}

Relationship between ratings of symmetry and rating of beauty.

Relationship between ratings of symmetry (salience) and rating of beauty. The regression lines match the way the statistical analyses have been carried out. In all graphs, the left panel is for the original stimuli and the right panel for stimuli with bilateral symmetry. The main graph at the top (A) shows all four categories using different colours. To highlight some important relationships the two additional panels combine some of the data. Panel B shows the overall link between salience and beauty. Panel $\mathrm{C}$ shows how this relationship is different in different categories and in particular for the nature stimuli (flowers and landscapes). 


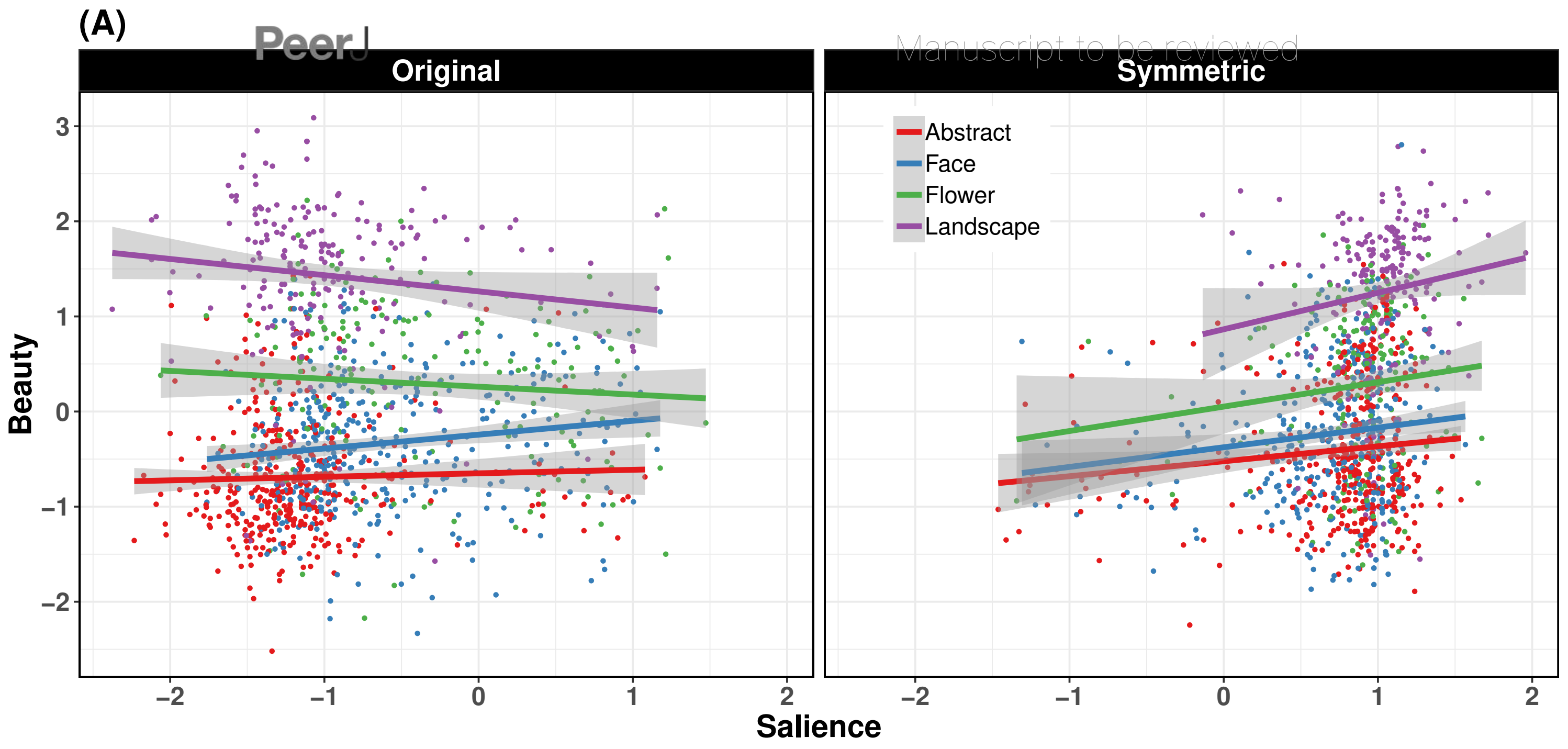

(B)

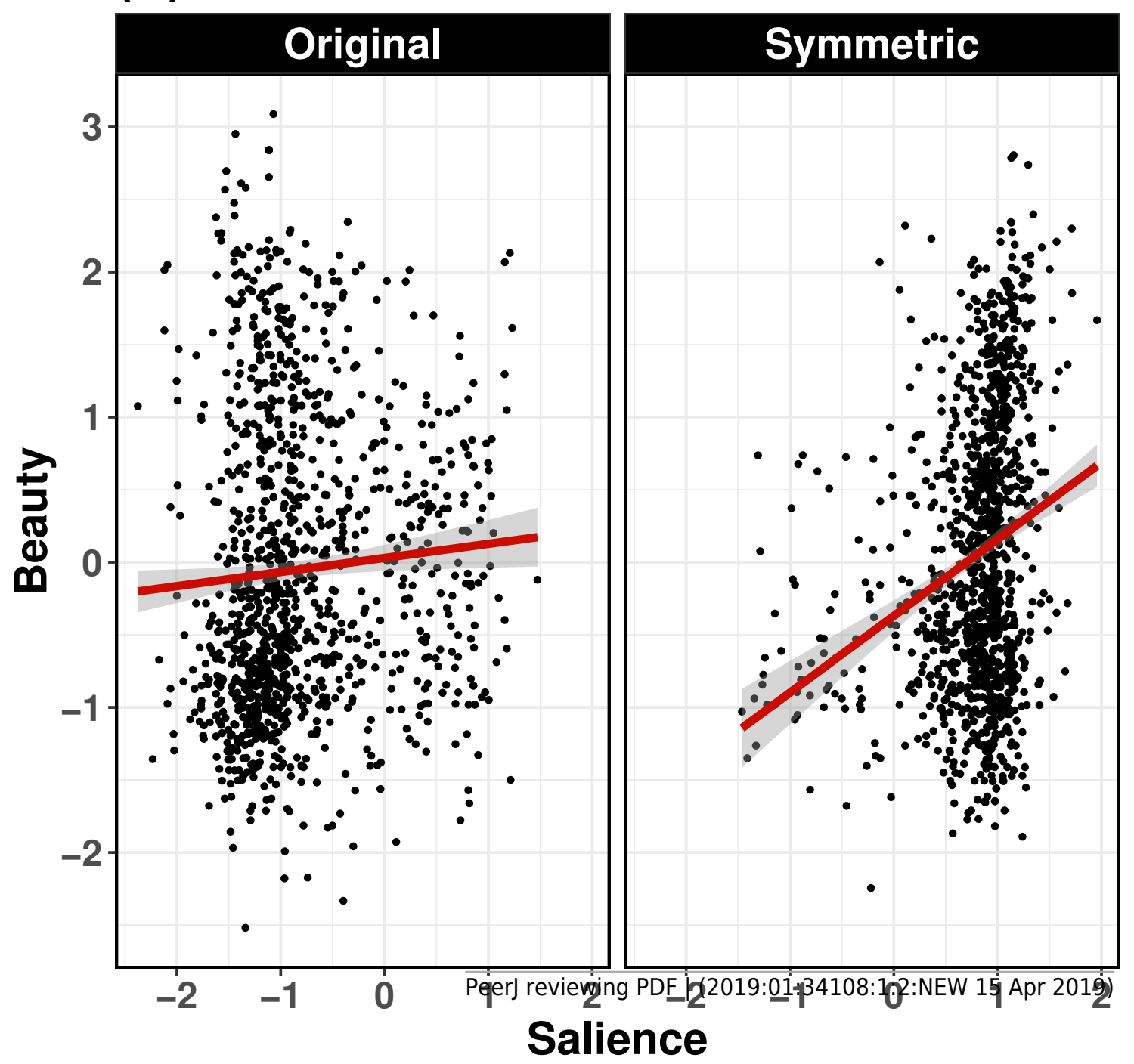

(C)

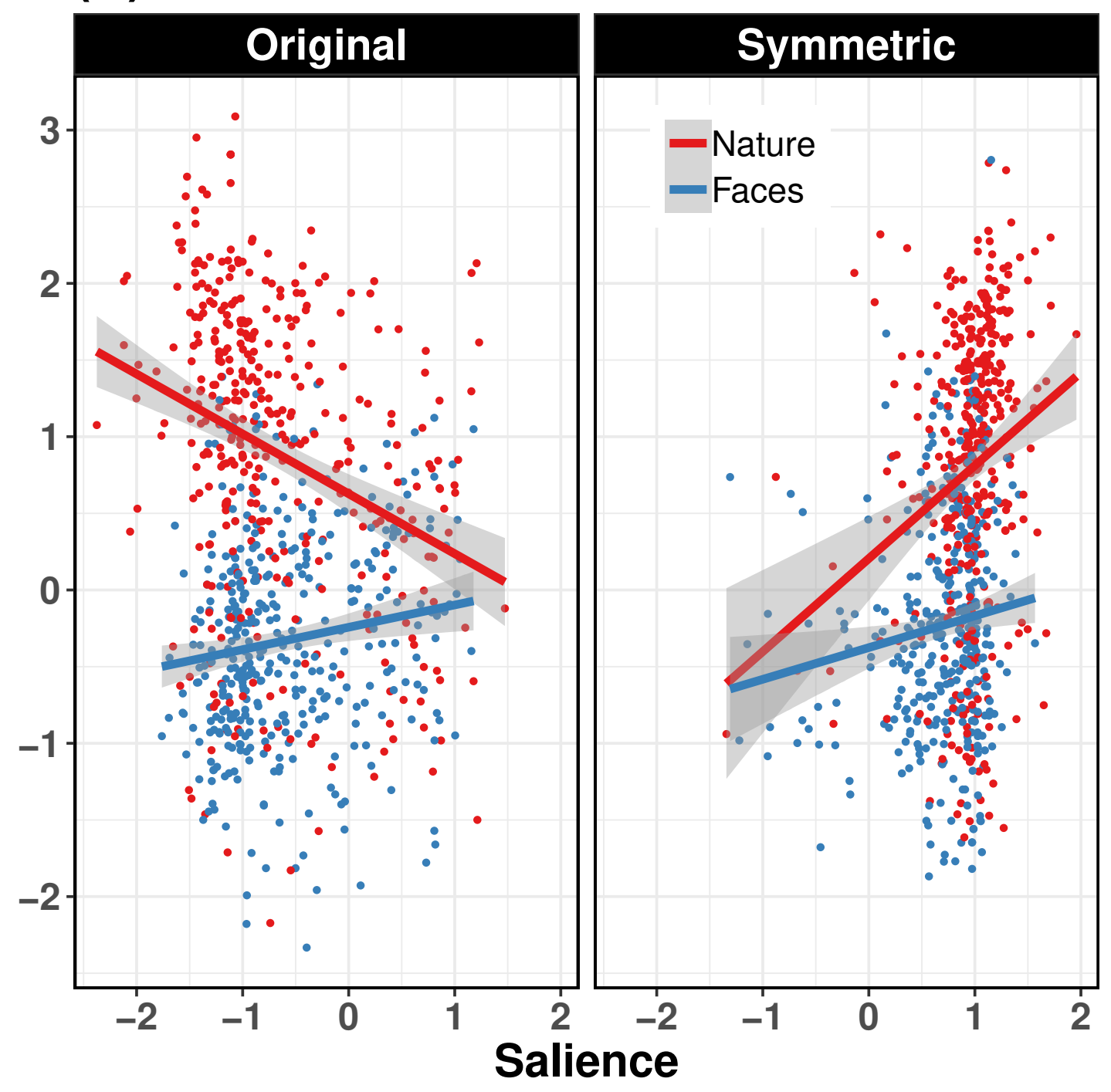


Figure 7 (on next page)

Relationship of preference in one category and preference for another category.

Scatterplot matrix to show how preference for symmetry for one category relates to preference for another category. Preference for bilateral symmetry is measured as the difference Symmetry - Original. Each point is an individual. People in the top right quadrants liked symmetry in both categories, people in the bottom left quadrants disliked symmetry in both categories, and in the other two quadrants we have inconsistent liking responses. 


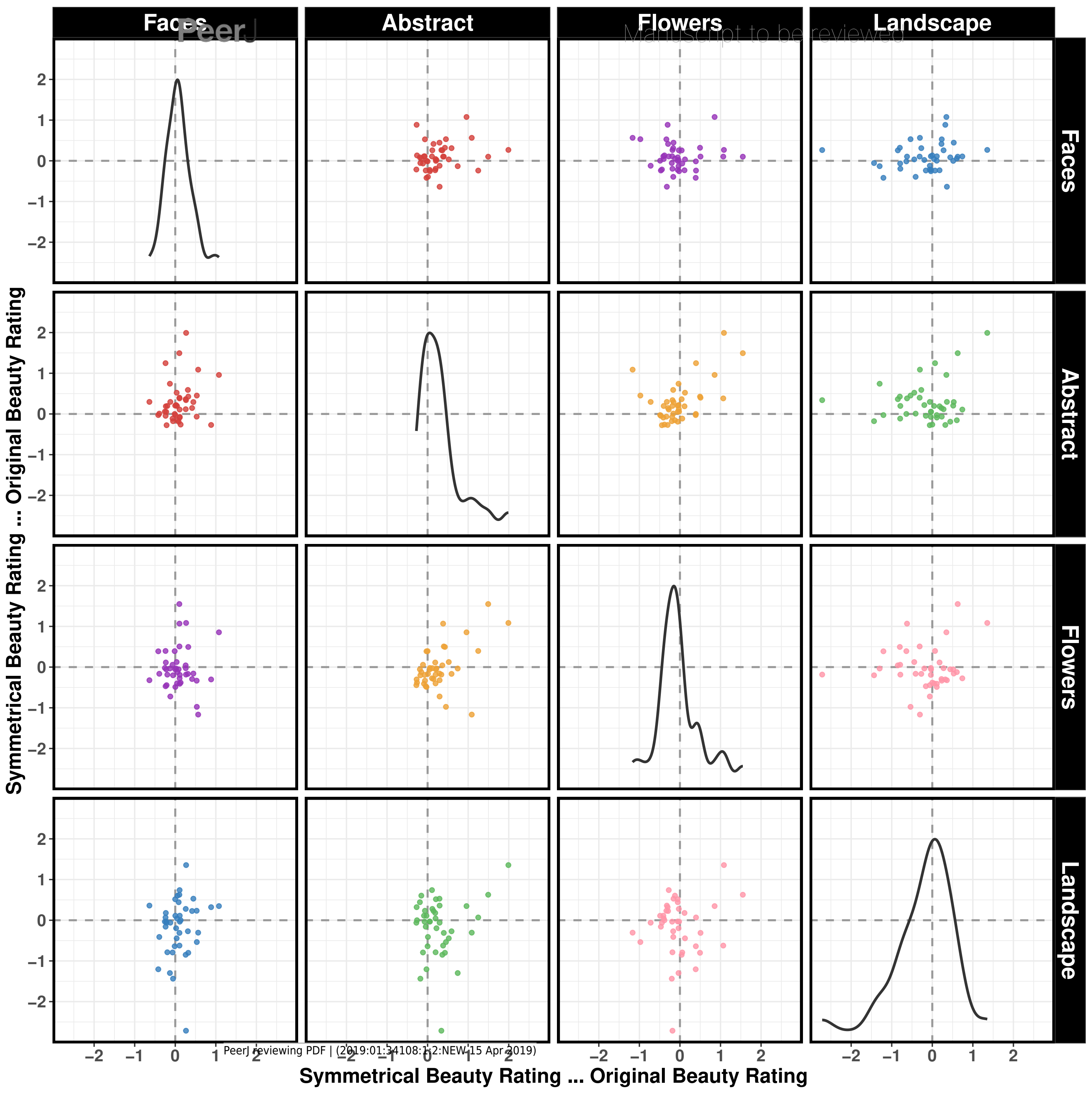




\section{Table 1 (on next page)}

Correlations for symmetry preference across participants.

Correlations for symmetry preference across participants. 
1 Table 1. Correlations for symmetry preference across participants.

\begin{tabular}{lllrrlc} 
Stimuli Type & Term & Beta & $\mathrm{SE}$ & $\mathrm{t}$ & $\mathrm{p}$ & \multicolumn{1}{c}{$R^{2}$} \\
\hline Faces & (Intercept) & $0.05[-0.08,0.18]$ & 0.07 & 0.69 & $=.492$ & 0.0703 \\
& Abstract & $0.18[-0.13,0.49]$ & 0.16 & 1.12 & $=.264$ & \\
& Flowers & $-0.08[-0.37,0.16]$ & 0.14 & -0.61 & $=.544$ & \\
\multirow{4}{*}{ Abstract } & Landscape & $0.06[-0.11,0.19]$ & 0.08 & 0.72 & $=.473$ & \\
& (Intercept) & $0.28[0.15,0.43]$ & 0.07 & 4.02 & $<.001$ & 0.2998 \\
& Faces & $0.28[-0.21,0.77]$ & 0.25 & 1.11 & $=.265$ & \\
& Flowers & $0.45[0.04,0.87]$ & 0.21 & 2.13 & $=.033$ & \\
& Landscape & $0.07[-0.14,0.3]$ & 0.11 & 0.61 & $=.545$ & \\
& (Intercept) & $-0.17[-0.28,-0.06]$ & 0.05 & -3.14 & $=.002$ & 0.2581 \\
& Abstract & $0.55[0.13,1.03]$ & 0.23 & 2.39 & $=.017$ & \\
& Faces & $-0.16[-0.67,0.36]$ & 0.26 & -0.61 & $=.544$ & \\
& Landscape & $0.03[-0.15,0.24]$ & 0.10 & 0.25 & $=.801$ & \\
& (Intercept) & $-0.24[-0.48,0.01]$ & 0.12 & -1.93 & $=.053$ & 0.0474 \\
& Abstract & $0.19[-0.4,0.87]$ & 0.32 & $0.60=.547$ & \\
& Faces & $0.25[-0.29,0.79]$ & 0.28 & 0.92 & $=.359$ & \\
& Flowers & $0.06[-0.34,0.55]$ & 0.23 & 0.26 & $=.793$ & \\
& & & & & &
\end{tabular}

